\title{
Dwarf novae in the Hamburg quasar survey: rarer than expected ${ }^{\star}$
}

\author{
A. Aungwerojwit ${ }^{1}$, B. T. Gänsicke ${ }^{1}$, P. Rodríguez-Gil ${ }^{1,2}$, H.-J. Hagen ${ }^{3}$, S. Araujo-Betancor ${ }^{2}$, \\ O. Baernbantner ${ }^{4}$, D. Engels ${ }^{3}$, R. E. Fried ${ }^{5}$, E. T. Harlaftis ${ }^{6}$, D. Mislis ${ }^{7}$, D. Nogami ${ }^{8}$, P. Schmeer ${ }^{9}$, \\ R. Schwarz ${ }^{10}$, A. Staude ${ }^{10}$, and M. A. P. Torres ${ }^{11}$ \\ 1 Department of Physics, University of Warwick, Coventry CV4 7AL, UK \\ e-mail: A. Aungwerojwit@warwick.ac.uk \\ 2 Instituto de Astrofísica de Canarias, 38200 La Laguna, Tenerife, Spain \\ 3 Hamburger Sternwarte, Universität Hamburg, Gojenbergsweg 112, 21029 Hamburg, Germany \\ 4 Universitäts-Sternwarte, Scheinerstr. 1, 81679 München, Germany \\ 5 Braeside Observatory, PO Box 906, Flagstaff AZ 86002, USA \\ ${ }^{6}$ Institute of Space Applications and Remote Sensing, National Observatory of Athens, PO Box 20048, Athens 11810, Greece \\ 7 Department of Physics, Section of Astrophysics, Astronomy \& Mechanics, University of Thessaloniki, 54124 Thessaloniki, \\ Greece \\ 8 Hida Observatory, Kyoto University, Kamitakara, Gifu 506-1314, Japan \\ 9 Bischmisheim, Am Probstbaum 10,66132 Saarbrücken, Germany \\ 10 Astrophysikalisches Institut Potsdam, An der Sternwarte 16, 14482 Potsdam, Germany \\ 11 Harvard-Smithsonian Center for Astrophysics, 60 Garden St, Cambridge, MA 02138, USA
}

Received 20 February 2006 / Accepted 11 May 2006

\section{ABSTRACT}

\begin{abstract}
Aims. We report the discovery of five new dwarf novae that were spectroscopically identified in the Hamburg Quasar Survey (HQS), and discuss the properties of the sample of new dwarf novae from the HQS.

Methods. Follow-up time-resolved spectroscopy and photometry have been obtained to characterise the new systems.

Results. The orbital periods determined from analyses of the radial velocity variations and/or orbital photometric variability are $P_{\text {orb }} \simeq 105.1 \mathrm{~min}$ or $P_{\text {orb }} \simeq 109.9 \mathrm{~min}$ for HS $0417+7445, P_{\text {orb }}=114.3 \pm 2.7 \mathrm{~min}$ for HS $1016+3412, P_{\text {orb }}=92.66 \pm 0.17 \mathrm{~min}$ for HS $1340+1524, P_{\text {orb }}=272.317 \pm 0.001 \mathrm{~min}$ for HS $1857+7127$, and $P_{\text {orb }}=258.02 \pm 0.56$ min for HS $2214+2845$. HS $1857+7127$ is found to be partially eclipsing. In HS $2214+2845$ the secondary star of spectral type M3 \pm 1 is clearly detected, and we estimate the distance to the system to be $d=390 \pm 40 \mathrm{pc}$. We recorded one superoutburst of HS $0417+7445$, identifying the system as a SU UMatype dwarf nova. HS $1016+3412$ and HS 1340+1524 have rare outbursts, and their subtype is yet undetermined. HS $1857+7127$ frequently varies in brightness and may be a Z Cam-type dwarf nova. HS 2214+2845 is a U Gem-type dwarf nova with a most likely cycle length of $71 \mathrm{~d}$.

Conclusions. To date, 14 new dwarf novae have been identified in the HQS. The ratio of short-period $(<3 \mathrm{~h})$ to long-period $(>3 \mathrm{~h})$ systems of this sample is 1.3 , much smaller compared to the ratio of 2.7 found for all known dwarf novae. The HQS dwarf novae display typically infrequent or low-amplitude outburst activity, underlining the strength of spectroscopic selection in identifying new CVs independently of their variability. The spectroscopic properties of short-period CVs in the HQS, newly identified and previously known, suggest that most, or possibly all of them are still evolving towards the minimum period. Their total number agrees with the predictions of population models within an order of magnitude. However, the bulk of all CVs is predicted to have evolved past the minimum period, and those systems remain unidentified. This suggests that those post-bounce systems have markedly weaker $\mathrm{H} \beta$ emission lines compared to the average known short-period CVs, and undergo no or extremely rare outbursts.
\end{abstract}

Key words. stars: dwarf novae - stars: individual: HS 0417+7445 - stars: individual: HS 1016+3412 stars: individual: HS 1340+1524 - stars: individual: HS 1857+7127 - stars: individual: HS 2214+2845

\section{Introduction}

Dwarf novae are a subset of non- (or weakly) magnetic cataclysmic variables $(\mathrm{CVs})$ that quasi-periodically brighten by several magnitudes. The commonly accepted cause for dwarf nova outbursts is a thermal instability in the accretion disc (e.g. Cannizzo et al. 1986; Osaki 1996). Within this theoretical framework, accretion discs undergo outbursts if the accretion rate is below a critical value, $\dot{M}_{\text {crit }}$. Above the $2-3 \mathrm{~h}$ period gap, the mass transfer rate in CVs is typically larger than $\dot{M}_{\text {crit }}$, and consequently only $\sim 36 \%$ of non-magnetic CVs with $P_{\text {orb }}>3 \mathrm{~h}$ are

* Figure 1 and Tables 2, 3 are only available in electronic form at http://www . edpsciences.org dwarf novae. For $P_{\text {orb }}<3 \mathrm{~h}$, the fraction of confirmed dwarf novae among the short-period non-magnetic $\mathrm{CVs}$ is $\sim 83 \%$. The large difference in mass transfer rate between short and long period systems is explained within the standard CV evolution theory by the cessation of magnetic braking - the dominant angular momentum loss mechanism in long period CVs - once the systems evolve down to $3 \mathrm{~h}$. Below $P_{\text {orb }}=3 \mathrm{~h}$, the evolution of CVs proceeds much slower due to lower mass transfer rates driven by gravitational radiation as the angular momentum loss mechanism (e.g. Rappaport et al. 1983; Spruit \& Ritter 1983; King 1988). For such low mass transfer rates, the disc instability model predicts thermally unstable accretion discs that produce dwarf-nova outbursts (Cannizzo 1993; Osaki 1996). Because of 
the long evolution time scale of low-mass transfer CVs population models built upon the disrupted magnetic braking scenario predict that $99 \%$ of all CVs should have periods below $3 \mathrm{~h}$ (Kolb 1993; Howell et al. 1997), which implies that the vast majority of all CVs is expected to be dwarf novae. Based on the current numbers, it appears that we know only a relatively small fraction of the predicted short-period CV population. Another prediction made by the population models is an accumulation of CVs near the minimum orbital period (e.g. Kolb \& Baraffe 1999), which is not observed. While this may signal a failure of the standard CV evolution theory (e.g. King et al. 2002; Barker \& Kolb 2003; Andronov et al. 2003), it is likely that the known sample of CVs is incomplete and biased. Dwarf novae are predominantly discovered because of their outbursts (Gänsicke 2005), and hence CVs with very long outburst recurrence times or low-amplitude outbursts could be substantially underrepresented in the known $\mathrm{CV}$ population.

As a measure to probe the completeness of the known CV sample, we have initiated a search based on one property common to the majority of all CVs: the presence of Balmer emission lines in their optical spectra. We are selecting CV candidates from the Hamburg Quasar Survey (HQS, Hagen et al. 1995), an objective prism Schmidt survey of the northern hemisphere covering $13600 \mathrm{deg}^{2}$ at high galactic latitudes with a limiting magnitude $17.5 \lesssim B \lesssim 18.5$. The survey resulted in $\simeq 50$ new CVs, including a number of peculiar objects (e.g. Gänsicke et al. 2000; Rodríguez-Gil et al. 2004b, 2005b; Araujo-Betancor et al. 2005a); a general overview has been given by Gänsicke et al. (2002) and more recently by Aungwerojwit et al. (2005).

In this paper, we report the identification of five new dwarf novae in the HQS: HS 0417+7445, HS 1016+3412, HS 1340+1524, HS 1857+7127, and HS 2214+2845 (HS 0417, HS 1016, HS 1340, HS 1857, and HS 2214, respectively, hereafter; Fig. 1 and Table 1). In Sect. 2 we provide details about the observations and data reduction, in Sects. 3-7 we describe the data analysis and determine the orbital periods of the new dwarf novae. In Sect. 8, we compare the period distribution of the dwarf novae found in the HQS to that of all known dwarf novae. In Sect. 9 we discuss the implications of our survey work on the space density of CVs.

\section{Observations and data reduction}

\subsection{Spectroscopy}

Identification spectra of HS 0417, HS 1016, HS 1340, HS 1857 , and HS 2214 were obtained at Calar Alto Observatory (Table 2). The spectra of all five systems (Fig. 2) contain strong Balmer emission lines on a blue continuum, together with weaker lines of He I that are characteristic of CVs. He II $\lambda 4686$ is very weak in all five systems, suggesting that they are dwarf novae observed in quiescence.

Additional time-resolved spectroscopy of HS 1016 (70 spectra), HS 1340 (78 spectra), HS 1857 (41 spectra), and HS 2214 (41 spectra) was obtained at the Calar Alto Observatory and Roque de los Muchachos Observatory (Table 2). The details of instrument setup and data reduction are described below.

Calar Alto Observatory. Identification spectroscopy and timeresolved spectroscopy were obtained with the Calar Alto Faint Object Spectrograph (CAFOS) at the $2.2 \mathrm{~m}$ telescope throughout the period October 1996 to February 2005 (Table 2), with the exception of HS 1857 which was identified as a CV in 1990 using the $\mathrm{B} \& \mathrm{C}$ spectrograph on the $2.2 \mathrm{~m}$ telescope. The identification spectra were obtained either with the $B-400$ or the $B-200$ grating through a $1.5^{\prime \prime}$ slit on a $2 \mathrm{k} \times 2 \mathrm{k}$ pixel SITe CCD and were reduced with the MIDAS quicklook context available at the Calar Alto.

The time-resolved follow-up observations of HS 1016, HS 1340, HS 1857, and HS 2214 were obtained with the $G-100$ grating and a $1.2^{\prime \prime}$ slit, providing a spectral resolution of $\sim 4.1 \AA$ (full width at half maximum, $F W H M$ ) over the wavelength range $\lambda \lambda 4240-8300$. Clouds and/or moderate to poor seeing affected a substantial fraction of these observations. HS 2214 was observed under photometric conditions using the $B-100$ grating along with a $1.5^{\prime \prime}$ slit, providing a resolution of $\sim 4 \AA(F W H M)$ over the range $3500-6300 \AA$. Two additional red spectra of HS 2214 were taken with the $R-100$ grating, covering the range $6000-9200 \AA$ at a similar resolution. All follow-up spectroscopy was obtained in $600 \mathrm{~s}$ exposures, interleaved with arc calibrations every $\sim 40 \mathrm{~min}$ to correct for instrument flexure. Flux standards were observed at the beginning and end of the night - weather permitting - to correct for the instrumental response. The data reduction (bias and flat-field correction, extraction, wavelength and flux calibration) was carried out using the Figaro package within Starlink and the programs Pamela and Molly developed by T. Marsh. Special care was given to the wavelength calibration by interpolating the dispersion relation for a given target spectrum from the two adjacent arc exposures.

Observatorio del Roque de los Muchachos. The Intermediate Dispersion Spectrograph (IDS) together with a $2 \mathrm{k} \times 4 \mathrm{k}$ pixel EEV10a detector was mounted at the $2.5 \mathrm{~m}$ Isaac Newton Telescope (INT) on La Palma to obtain time-resolved spectroscopy of HS 1016, HS 1340, HS 1857, and HS 2214 (Table 2). The R632V grating and a slit width of $1.5^{\prime \prime}$ provided a spectral resolution of $\sim 2.3 \AA(F W H M)$ and a useful wavelength range of $\sim 4400-6800 \AA$. The data reduction was carried out along the same lines as described above for the Calar Alto data using IRAF $^{1}$ and Molly.

\subsection{Photometry}

Throughout the period December 2000 to May 2005, we obtained time-series differential CCD photometry of the five new dwarf novae during a total of 54 nights using ten different telescopes. The individual objects were observed for $\sim 25 \mathrm{~h}$ (HS 0417), 14h (HS 1016), 68 h (HS 1340), 50 h (HS 1857), and $\sim 48 \mathrm{~h}$ (HS 2214). Sample light curves are shown in Figs. 3 to 7 . The details of the observations and instruments used are given in Table 2. The data obtained at Wendelstein, Calar Alto, Kryoneri, INT, and OLT were reduced using the pipeline described by Gänsicke et al. (2004a), which uses the Sextractor (Bertin \& Arnouts 1996) to calculate aperture photometry for all objects in the field of view. The AIP data were reduced entirely within MIDAS. Bias and flat-field correction of the OGS, IAC80, and FLWO images as well as the extraction of Point Spread Function (PSF) magnitudes were done using IRAF. For the Braeside data, the reduction was performed in a standard way using a custom-made software suite. Finding charts of all five dwarf novae are shown in Fig. 1. The comparison stars used in the reduction of our differential CCD photometry are listed

\footnotetext{
1 IRAF is distributed by the National Optical Astronomy Observatories.
} 
Table 1. Properties of the five new dwarf novae.

\begin{tabular}{lccccc}
\hline \hline & HS $0417+7445$ & HS $1016+3412$ & HS 1340+1524 & HS 1857+7127 & HS 2214+2845 \\
\hline Right ascension $(\mathrm{J} 2000)$ & $04^{\mathrm{h}} 23^{\mathrm{m}} 32.8^{\mathrm{s}}$ & $10^{\mathrm{h}} 19^{\mathrm{m}} 47.3^{\mathrm{s}}$ & $13^{\mathrm{h}} 43^{\mathrm{m}} 23.2^{\mathrm{s}}$ & $18^{\mathrm{h}} 57^{\mathrm{m}} 20.4^{\mathrm{s}}$ & $22^{\mathrm{h}} 16^{\mathrm{m}} 31.2^{\mathrm{s}}$ \\
Declination $(\mathrm{J} 2000)$ & $+74^{\circ} 52^{\prime} 50.30^{\prime \prime}$ & $+33^{\circ} 57^{\prime} 53.9^{\prime \prime}$ & $+15^{\circ} 09^{\prime} 16.9^{\prime \prime}$ & $+71^{\circ} 31^{\prime} 19.2^{\prime \prime}$ & $+29^{\circ} 00^{\prime} 20.6^{\prime \prime}$ \\
Period (min) & $\simeq 105.1 / \simeq 109.9$ & $114.3 \pm 2.7$ & $92.66 \pm 0.17$ & $272.317 \pm 0.001$ & $258.02 \pm 0.56$ \\
Magnitude range & $18.0-13.5$ & $18.6-15.4:$ & $18.5-14.2$ & $17.2-13.9$ & $16.5-12.3$ \\
$\mathrm{H} \alpha E W[\AA] / F H W M[\AA]$ & $172 / 43$ & $184 / 27$ & $121 / 28$ & $39 / 32$ & $53 / 33$ \\
$\mathrm{H} \beta E W[\AA] / F H W M[\AA]$ & $98 / 43$ & $125 / 25$ & $88 / 23$ & $33 / 32$ & $42 / 31$ \\
$\mathrm{H} \gamma E W[\AA] / F H W M[\AA]$ & $73 / 38$ & $85 / 24$ & $59 / 22$ & $27 / 33$ & $30 / 32$ \\
He I $\lambda 5876 E W[\AA] / F H W M[\AA]$ & $40 / 43$ & $52 / 31$ & $36 / 30$ & $7 / 34$ & $7 / 25$ \\
He I $\lambda 6678 E W[\AA] / F H W M[\AA]$ & $7 / 30$ & $26 / 35$ & $18 / 32$ & $3 / 46$ & $5 / 39$ \\
RASS source $(1 \mathrm{RXS} \mathrm{J})$ & $042332.8+745300$ & $101946.7+335811$ & $134323.1+150916$ & $185722.6+713126$ & $221631.2+290025$ \\
RASS count rate $\left(0.01 \mathrm{cts} \mathrm{s}^{-1}\right)$ & $6.0 \pm 1.3$ & $2.5 \pm 1.0$ & $7.3 \pm 1.7$ & $3.4 \pm 4.5$ & $9.9 \pm 1.5$ \\
Hardness ratio HR1 & $1.00 \pm 0.09$ & $1.00 \pm 0.91$ & $0.18 \pm 0.23$ & $1.00 \pm 0.04$ & $0.92 \pm 0.06$ \\
Hardness ratio HR2 & $0.21 \pm 0.20$ & $-0.32 \pm 0.38$ & $-0.05 \pm 0.27$ & $0.69 \pm 0.10$ & $0.10 \pm 0.14$ \\
\hline
\end{tabular}

Notes. The coordinates are taken from the USNO-B catalogue (Monet et al. 2003); the ROSAT PSPC count rates and hardness ratios HR1 and HR2 have been obtained from the ROSAT All Sky Survey (RASS) Bright Source Catalogue (Voges et al. 1999) and from the RASS Faint Source Catalogue (Voges et al. 2000); the $\mathrm{H} \alpha-\mathrm{H} \gamma$ and $\mathrm{He}$ I $\lambda 5876,6678$ equivalent widths $(E W)$ and full width at half maximum $(F W H M)$ were measured from the Calar Alto average spectra (Fig. 2) using the integrate/line task in MIDAS; the outburst of HS 1016 is uncertain (marked by a colon), as only one outburst was observed.

in the last column of Table 2 (see Fig. 1 for identifications), and their USNO $R$ and $B$ magnitudes are given in Table 3 .

Additional images of HS 0417, HS 1016, HS 1340, and HS 2214 were taken intermittently during the period May 2004 to April 2005 using the $0.37 \mathrm{~m}$ robotic Rigel telescope of the University of Iowa which is equipped with a $1 \mathrm{k} \times 1 \mathrm{k}$ pixel SITe003 CCD camera. For all four systems, filterless images with an exposure time of $25 \mathrm{~s}$ were obtained.

\subsection{The new dwarf novae as $X$-ray sources}

All five dwarf novae identified on the basis of their emission line spectra in the HQS are also X-ray sources in the ROSAT All Sky Survey (RASS): HS 0417, HS 1340, and HS 2214 are contained in the Bright Source Catalogue (Voges et al. 1999), HS 1016 and HS 1857 within the Faint Source Catalogue (Voges et al. 2000). The X-ray properties of the new systems are summarised in Table 1. All but HS 1340 are hard X-ray sources in the hardness ratio HR1, typical of non- (or weakly-) magnetic CVs (van Teeseling et al. 1996).

\section{HS $0417+7445$}

Our identification spectrum of HS 0417 obtained in October 1996 (Fig. 2, Table 2) is dominated by low-excitation emission lines, typical of a dwarf nova. HS 0417 is contained in the ROSAT Bright Source Catalogue as 1RXS J042332+745300 (Voges et al. 1999), and has been independently identified as a CV by Wu et al. (2001). HS 0417 displayed large-amplitude variability on the HQS spectral plates, where it was detected at $B \simeq 18.0$ in June 1992 and at $B \simeq 13.7$ in October 1995, supporting the suggested dwarf nova nature of the object.

Throughout our photometric observations we have found the object near a mean magnitude of $\simeq 17.5$ (December 2000: $B \simeq$ 17.9, February 2003: $R \simeq 17.3$, November 2004: filterless $\simeq 17.6$, January 2005: $g^{\prime} \simeq 17.5$ ), consistent with the USNO-A2.0 measurements, $R \simeq 17.2$ and $B \simeq 16.8$, except during January 2001, when the system was found in an outburst near $B \simeq 13.5$. In the quiescent state, the light curve of HS 0417 is characterised by a double-humped pattern with a period of $\sim 100$ min (Fig. 3, bottom panel). The light curve obtained during the January 2001 outburst (Fig. 3, top panel) reveals superhumps that identify HS 0417 as a SU UMa-type dwarf nova and therefore this outburst as a superoutburst. An additional outburst of HS 0417 was caught on the rise in April 10, 2005 by one of us (PS), and $\sim 3 \mathrm{~h}, V$-band data obtained by David Boyd on the evening of April 11, 2005 showed the object already declining at a rate of $\sim 0.85 \mathrm{mag} \mathrm{d}^{-1}$ and no evidence of superhumps was found. By April 18, the system reached again its quiescent magnitude of $V \simeq 17.5$.

In order to measure the orbital period of the system, a Scargle (1982) periodogram was computed within the MIDAS/TSA context from all quiescent data except the February 2003 observations which were of too poor a quality. The periodogram (Fig. 8) contains a fairly broad sequence of aliases spaced by $1 \mathrm{~d}^{-1}$ with the strongest signal at $13.7 \mathrm{~d}^{-1}$ and a nearly equally strong signal at $13.1 \mathrm{~d}^{-1}$. The high-frequency range of the periodogram of HS 0417 is nicely reproduced by the window function (shifted to $13.7 \mathrm{~d}^{-1}$ in the top panel of Fig. 8), but excess power is present at frequencies below $10 \mathrm{~d}^{-1}$, most likely associated with the short length of the observing runs. Sine-fits to the data result in the periods corresponding to the two highest peaks in the periodogram, $P \simeq 105.1 \mathrm{~min}$ and $P \simeq 109.9 \mathrm{~min}$, respectively. We interpreted these values as possible orbital periods of HS 0417 .

The Scargle periodogram computed from the superoutburst data obtained on January 14, 2001 (Fig. 3, top panel) provides a broad signal with a peak at $\simeq 13.3 \mathrm{~d}^{-1}$, or $P \simeq 108.3 \mathrm{~min}$. The light curve folded over this period shows, however, a significant offset between the two observed superhump maxima. A periodogram computed using Schwarzenberg-Czerny's (1996) analysis-of-variance (AOV) method using orthogonal polynomial fits to the data (implemented as ORT/TSA in MIDAS) results in a much narrower peak compared to the Scargle analysis, centred at $12.95 \mathrm{~d}^{-1}(P \simeq 111.2 \mathrm{~min})$. This period provides a clean folded light curve. This improvement in the period analysis underlines the fact that AOV-type methods provide better sensitivity for strongly non-sinusoidal signals (such as superhumps) compared to Fourier-transform based methods.

The analysis of our photometric data left us with two candidate orbital periods, $P_{\text {orb }}=105.1 \mathrm{~min}$ or $P_{\text {orb }}=109.9 \mathrm{~min}$, and two candidate superhump periods, $P_{\mathrm{sh}}=108.3 \mathrm{~min}$ or 


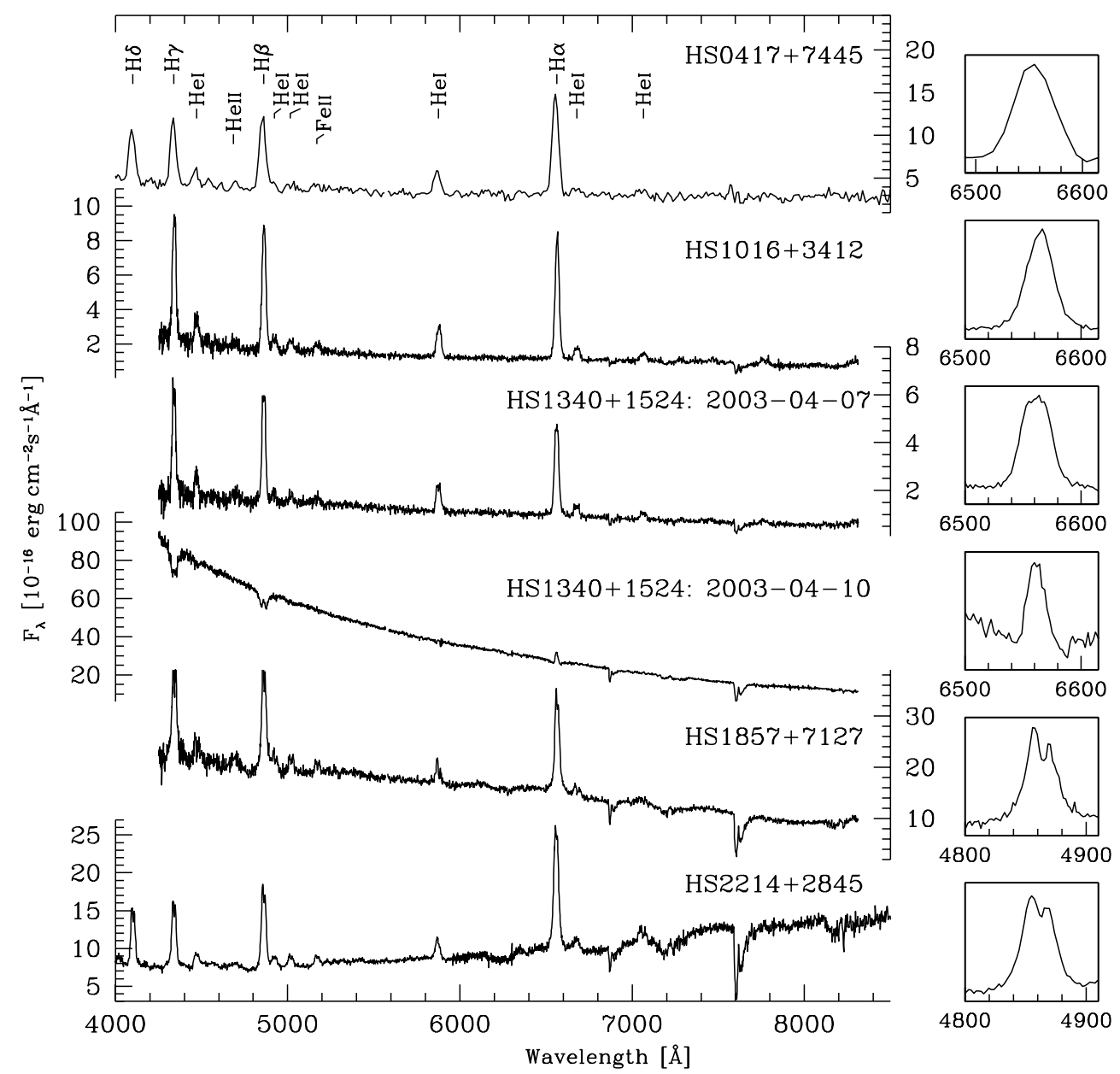

Fig. 2. Main panel: flux-calibrated CAFOS spectra of HS 0417, HS 1016, HS 1340, HS 1857, and HS 2214. Fluxes are labelled alternatingly on the left and right side. HS 1340 was observed in quiescence and outburst, respectively. Right panel: close-up plots of the $\mathrm{H} \alpha$ and $\mathrm{H} \beta$ profiles.
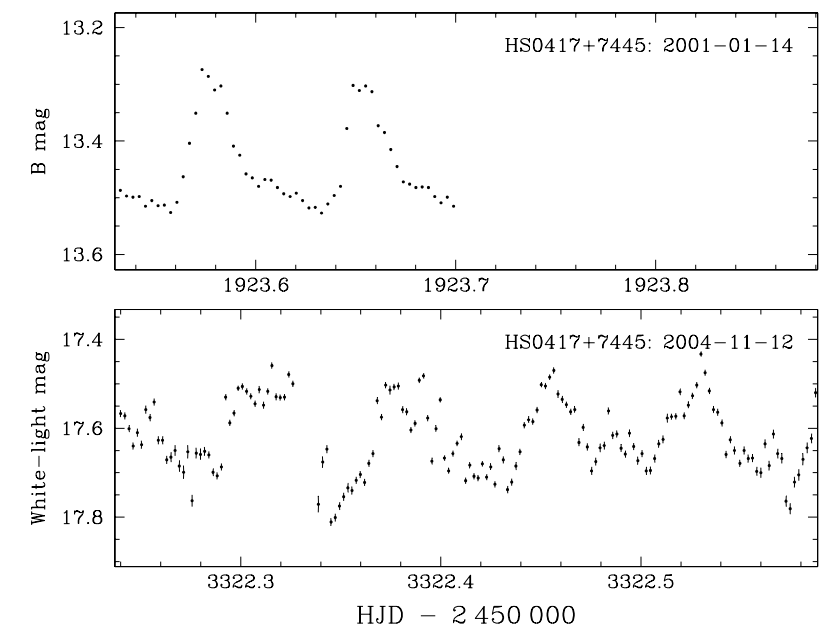

Fig. 3. Sample light curves of HS 0417 obtained at the Wendelstein observatory. Top panel: $B$-band data obtained during superoutburst on January 14, 2001. Bottom panel: filterless data obtained during quiescence.

$P_{\text {sh }}=111.2 \mathrm{~min}$. Table 4 lists the fractional superhump excess, $\epsilon=\left(P_{\text {sh }}-P_{\text {orb }}\right) / P_{\text {orb }}$ calculated from all possible combinations of the candidate periods. We consider cases (2) and (3) as very unlikely, as no dwarf nova with $\epsilon>5 \%$ is found below the period gap and no short-period dwarf nova with a negative
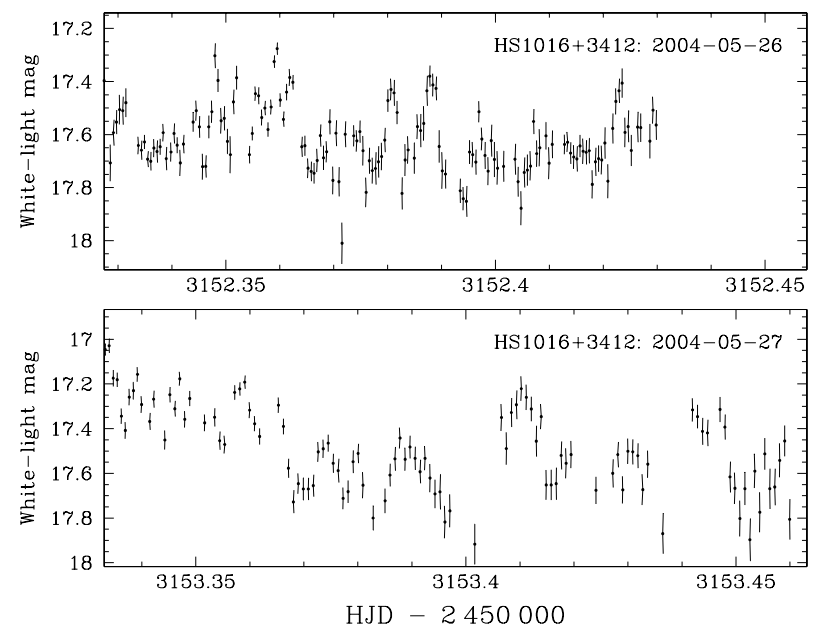

Fig. 4. Sample filterless light curves of HS 1016 obtained at the Kryoneri observatory.

superhump excess is known (e.g. Nogami et al. 2000; Patterson et al. 2003; Rodríguez-Gil et al. 2005a). In fact, most dwarf novae with $P_{\text {orb }} \sim 100-115$ min have $\epsilon \sim 3-4 \%$ (Patterson et al. 2005), which would make case (1) look most likely. However, based on our data, we prefer case (4) as $P_{\mathrm{sh}}=111.2 \mathrm{~min}$ gave the cleanest folded superhump light curve. In this case, HS 0417 would have a rather low value of $\epsilon$, similar only to KV And 

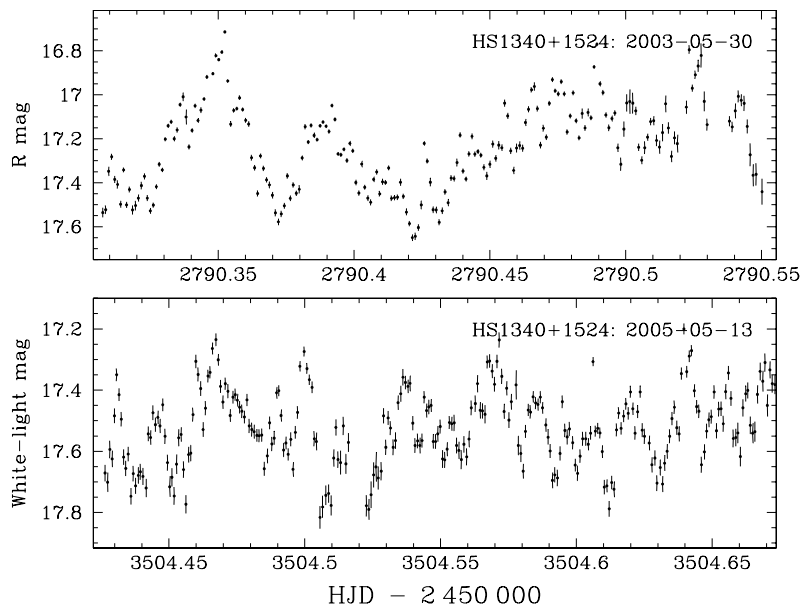

Fig. 5. Sample light curves of HS 1340. Top panel: $R$-band data obtained at the Kryoneri observatory. Bottom panel: filterless data obtained at the IAC80 telescope.
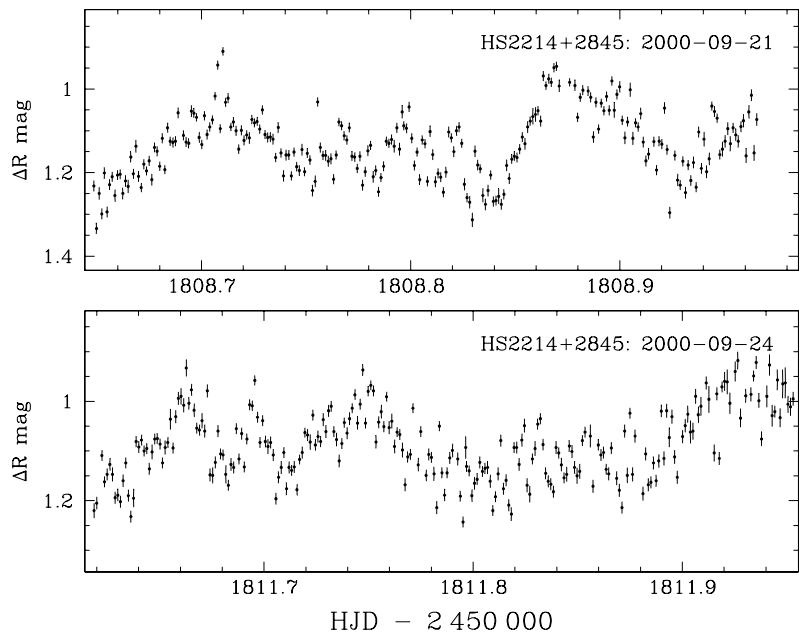

Fig. 6. Sample $R$-band light curves of HS 2214 obtained at the Braeside observatory.

Table 4. The fractional superhump excess of HS 0417 computed from $\epsilon=\left(P_{\text {sh }}-P_{\text {orb }}\right) / P_{\text {orb }}$.

\begin{tabular}{cccc}
\hline \hline Case & $P_{\text {orb }}(\min )$ & $P_{\text {sh }}(\min )$ & $\epsilon$ \\
\hline 1 & 105.1 & 108.3 & 0.030 \\
2 & 105.1 & 111.2 & 0.058 \\
3 & 109.9 & 108.3 & -0.015 \\
4 & 109.9 & 111.2 & 0.012 \\
\hline
\end{tabular}

$\left(P_{\text {orb }}=105.49(30)\right.$ min) which has $\epsilon=0.0145$ (Patterson et al. 2003). An unambiguous determination of both $P_{\text {orb }}$ and $P_{\text {sh }}$ would be important, as $\epsilon$ may be used to estimate the mass ratio of a CV (Patterson et al. 2005).

\section{HS $1016+3412$}

The CAFOS (Fig. 2) and INT average spectra of HS 1016 are similar to that of HS 0417, with strong Balmer emission lines together with weaker $\mathrm{He}_{\mathrm{I}}$ and $\mathrm{Fe}$ II lines and practically absent He II 14686 . Our photometric time-series (Table 2) found the system consistently at a magnitude of $\simeq 17.5$. The system was found fainter, $V \simeq 18.6$, in the April 2003 CAFOS acquisition

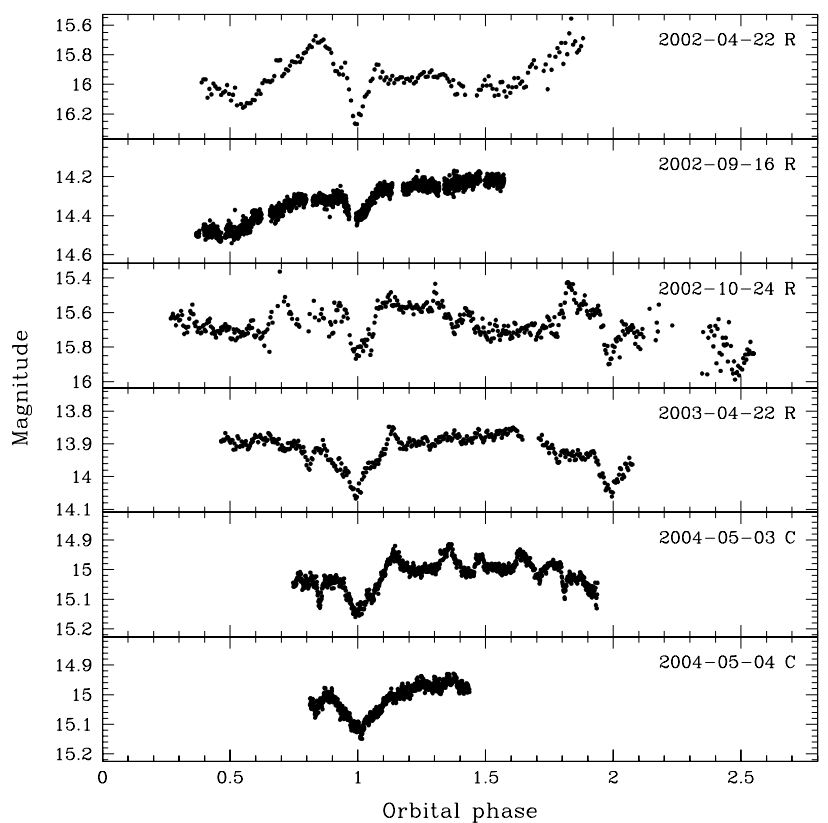

Fig. 7. Sample $R$-band and filterless light curves of HS 1857 folded over the ephemeris in Eq. (1). See Sect. 6.2 for details.

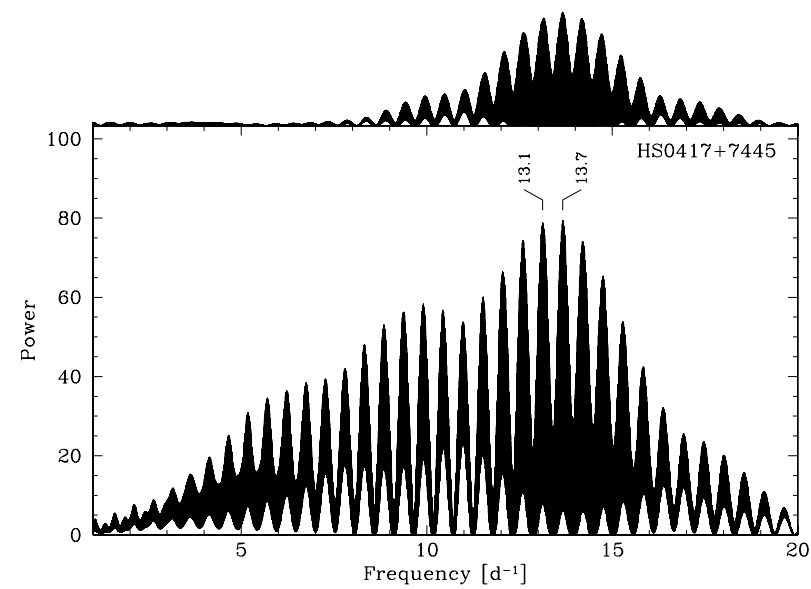

Fig. 8. Main panel: the Scargle periodogram of HS 0417 during quiescence computed from all photometric data except February 27, 2003. Top panel: the window function shifted to $13.7 \mathrm{~d}^{-1}$.

images. The only known outburst of HS 1016 was detected using the Rigel telescope on November 2, 2004, where an unfiltered magnitude of 15.4 was recorded. The next image obtained on November 11 showed the system again at its quiescent magnitude of $\simeq 17.5$.

The single-peaked profile found in the emission lines suggests a relatively low orbital inclination. No spectral contribution from the secondary star is detected in the red part of the spectrum. The equivalent widths $(E W s)$ from the CAFOS and INT average spectra do not show any noticeable variation in each epoch throughout our run. Table 1 lists FWHM and EW parameters of the CAFOS average spectrum measured from Gaussian fits.

In order to determine the orbital period of HS 1016, we measured the radial velocity variation of $\mathrm{H} \alpha$, the strongest emission line, from the CAFOS and IDS spectra. We first rebinned the individual spectra to a uniform velocity centred on $\mathrm{H} \alpha$, followed by normalising the slope of the continuum. We then measured the $\mathrm{H} \alpha$ radial velocity variation using the double 


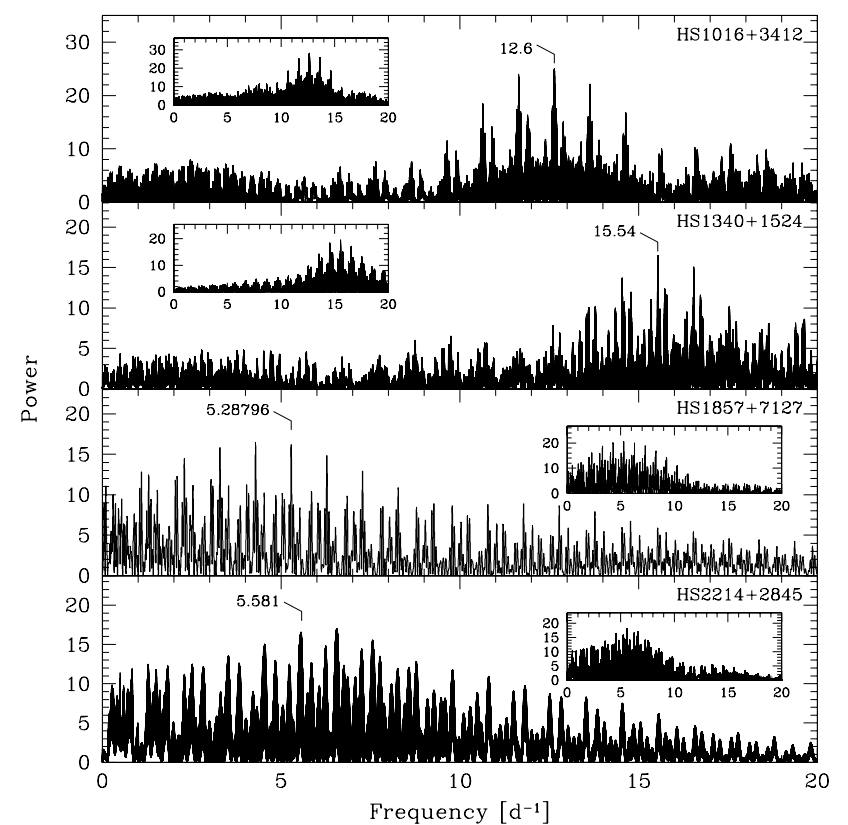

Fig. 9. The Scargle periodogram of the radial velocities of HS 1016, HS 1340, HS 1857, and HS 2214. The periodograms constructed from faked sets of data at the corresponding orbital frequency are shown in small windows.

Gaussian method of Schneider \& Young (1980) with a separation of $1000 \mathrm{~km} \mathrm{~s}^{-1}$ and an $F W H M$ of $200 \mathrm{~km} \mathrm{~s}^{-1}$. A Scargle periodogram calculated from the $\mathrm{H} \alpha$ radial velocity variation contains a set of narrow aliases spaced by $1 \mathrm{~d}^{-1}$, with the strongest signal found at $f \simeq 12.6 \mathrm{~d}^{-1}$ (Fig. 9, top panel). We tested the significance of this signal by creating a faked set of radial velocities computed from a sine function with a frequency of $12.6 \mathrm{~d}^{-1}$, and randomly offset from the computed sine wave using the observed errors. The periodogram of the faked data set is plotted in a small window of the top panel in Fig. 9 which reproduces well the alias structure of the periodogram calculated from the observation. A sine-fit to the folded radial velocities refined the period to $114.3 \pm 2.7 \mathrm{~min}$, which we interpreted as the orbital period of HS 1016. Figure 10 (top panel) shows a sine-fit to the phasefolded radial velocity curve; the fit parameters are reported in Table 5.

The light curves of HS 1016 display short-time scale flickering with an amplitude of $\sim 0.2-0.3 \mathrm{mag}$ (Fig. 4). A Scargle periodogram computed from the entire photometry as well as from individual subsets did not reveal any significant signal.

\section{HS 1340+1524}

The average spectrum of HS 1340 during quiescence (Fig. 2) is similar to that of HS 0417 and HS 1016, showing strong singlepeaked line profiles of Balmer emissions along with the weaker lines of $\mathrm{He}$ I and $\mathrm{Fe}$ II. The line parameters during quiescence are given in Table 1.

\subsection{Long and short term variability}

Throughout our time series photometry obtained at the AIP, IAC80, FLWO, and Kryoneri, HS 1340 was found at a mean magnitude in the range $\sim 17.7-16.8$ (see Fig. 11, main window). A first outburst of HS 1340 was detected on the evening of April 10, 2003 during observations with the $2.2 \mathrm{~m}$ telescope at

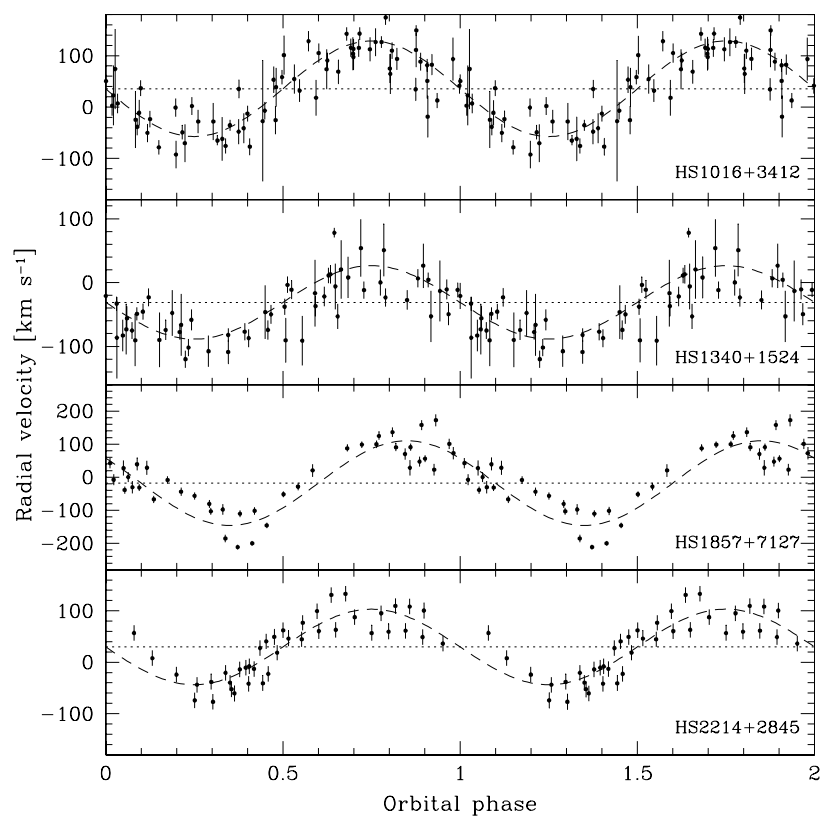

Fig. 10. $\mathrm{H} \alpha$ radial velocities of HS 1016, HS 1340, and HS 1857 and $\mathrm{H} \alpha$ plus $\mathrm{H} \beta$ radial velocities of HS 2214. The radial velocities of HS 1016 and HS 1340 are folded on their spectroscopic periods of $114.3 \mathrm{~min}$ and $92.66 \mathrm{~min}$, respectively and those of HS 1857 and HS 2214 are folded on their photometric periods of $272.317 \mathrm{~min}$ and $258.02 \mathrm{~min}$, respectively. The data on HS 1857 were folded using the eclipse ephemeris given in Eq. (1) while the other three systems were folded defining phase zero as the time of red-to-blue crossing of the radial velocities.

the Calar Alto Observatory (Table 2). The outburst peak magnitude was $V \simeq 14.7$ on the CAFOS acquisition image. The spectra obtained immediately thereafter show weak emission at $\mathrm{H} \alpha$, with an equivalent width of $\sim 4.7 \AA$, whereas $\mathrm{H} \beta$ and $\mathrm{H} \gamma$ are in absorption with narrow emission cores, which are typical of an optically thick accretion disc. Similar spectra were observed in the three HQS CVs: HS 0139+0559, HS 0229+8016, and HS 0642+5049 (Aungwerojwit et al. 2005). As the conditions during the night deteriorated, we switched to time-series photometry, recording a decline at $\sim 0.05 \mathrm{mag} \mathrm{h}^{-1}$. The CAFOS acquisition images showed that HS 1340 faded to $V \simeq 16.1$ and $V \simeq 17.3$ in the two subsequent nights, April 11 and 12, 2003, respectively. A puzzling fact is that acquisition images taken before the outburst on April 7, 8, and 9, 2003 showed HS 1340 at $V \simeq 18.5$, i.e. nearly one magnitude fainter than the usual quiescent value (see Fig. 11, small window). On April 28, a CAFOS acquisition image showed the system again at a filterless magnitude of 17.6, consistent with the typical quiescent brightness. The duration of the entire outburst was less than two days.

A second outburst reaching an unfiltered magnitude of $\sim 14.2$ was recorded on April 15, 2005 with the Rigel telescope, again, the duration of the outburst was of the order of 2-3 days.

The light curves of HS 1340 obtained during quiescence are predominantly characterised by variability on time scales of 15-20 min with peak-to-peak amplitudes of $\sim 0.4$ mag (Fig. 5, bottom panel). On some occasions, the light curves shows humplike structures which last for one to several hours, superimposed by short-time scale flickering (e.g. Fig. 5, top panel). Our period analysis of the photometric data did not reveal any stable signal in the combined data.

In summary, HS 1340 appears to have rather infrequent and short-lived outbursts, and displays a substantial amount of 
Table 5. Sine fits to the $\mathrm{H} \alpha$ radial velocities of HS 1016, HS,1340, and HS 1857. For HS 2214 a combination of $\mathrm{H} \alpha$ and $\mathrm{H} \beta$ radial velocities were fitted, as the September 2000 Calar Alto spectra did not cover H $\alpha$. For HS 1857 and HS 2214, the periods were fixed to their values determined from the photometry.

\begin{tabular}{ccccc}
\hline \hline Object & $T_{0}$ & Period (days) & $K\left(\mathrm{~km} \mathrm{~s}^{-1}\right)$ & $\gamma\left(\mathrm{km} \mathrm{s}^{-1}\right)$ \\
\hline HS 1016+3412 & $2452737.4039 \pm 0.0012$ & $0.0794 \pm 0.0019$ & $93.0 \pm 5.3$ & $35.6 \pm 3.9$ \\
HS 1340+1524 & $2452737.4438 \pm 0.0018$ & $0.06435 \pm 0.00012$ & $57.5 \pm 6.2$ & $-31.1 \pm 4.1$ \\
HS 1857+7127 & $2452368.53243 \pm 0.00098$ & $0.189109 \pm 0.000001$ & $128.0 \pm 9.6$ & $-17.9 \pm 7.4$ \\
HS 2214+2845 & $2451812.3309 \pm 0.0028$ & $0.17918 \pm 0.00039$ & $73.5 \pm 6.5$ & $29.8 \pm 5.0$ \\
\hline
\end{tabular}

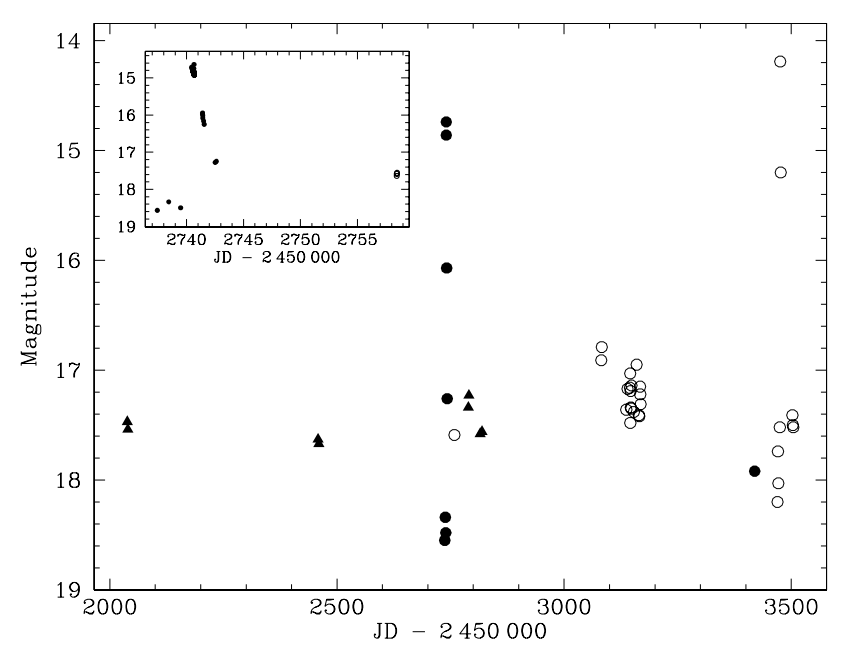

Fig. 11. Main window: the mean magnitudes of HS1340 obtained from May 2001 to May 2005 in $R$-band (filled triangles), $V$-band (filled circles), and white light (open circles). The photometric error on the individual points is $<0.05 \mathrm{mag}$. An additional systematic uncertainty arises from the combination of different band passes. Considering the apparent magnitudes of HS 1340 listed in the Sloan Digital Sky Survey which are $g=17.3, r=17.1$, and $i=17.1$, the errors due to colour terms are likely to be within \pm 0.1 mag. Small window: close up of the April 2003 CAFOS run. The first three points show the deep faint state with a mean magnitude of $V \simeq 18.5$ before the outburst on April 10, 2003.

short-term variability as well as variability of its mean magnitude during quiescence.

\subsection{The orbital period}

The orbital period of HS 1340 was determined using the spectroscopic data taken in quiescence. The $\mathrm{H} \alpha$ radial velocity variation was measured in the same manner as in HS 1016 with a separation of $1000 \mathrm{~km} \mathrm{~s}^{-1}$ and an $F W H M$ of $200 \mathrm{~km} \mathrm{~s}^{-1}$. Figure 9 (second panel) shows the Scargle periodogram. The strongest signal is found at $f=15.54 \pm 0.03 \mathrm{~d}^{-1}$ where the error is estimated from the $F W H M$ of the strongest peak in the periodogram, corresponding to an orbital period of $P_{\text {orb }}=92.66 \pm 0.17 \mathrm{~min}$. The $\mathrm{H} \alpha$ radial velocity curve folded over this period is shown in Fig. 10 (second panel) along with a sine-fit; the fit parameters are given in Table 5. The periodogram of a faked data set constructed from this frequency agrees well with the entire observed alias structure (insert in Fig. 9, second panel).

With the spectroscopic period being determined, we reanalysed the time-series photometry of HS 1340, and found no significant signal in the range of the orbital frequency when we combined all quiescent data. However, a weak signal at a frequency of $\sim 15.5 \mathrm{~d}^{-1}$ and its one-day aliases were detected intermittently on some occasions, e.g. in the 2003 Kryoneri data and the 2004 FLWO observations.

\section{HS $1857+7127$}

The CAFOS average spectrum of HS 1857 (Fig. 2) is similar to the spectra of HS 0417, HS 1016, and HS 1340, presenting a blue slope superimposed by Balmer and $\mathrm{He}_{\mathrm{I}}$ emission lines. Slight flux depressions are observed near $6200 \AA$ and $7200 \AA$, which might be associated with the $\mathrm{TiO}$ bands of an M-type donor, however, the quality of the data is insufficient to unambiguously establish the detection of the secondary star. The Balmer emission line profiles are double-peaked, with a peak-to-peak separation of $\sim 800 \mathrm{~km} \mathrm{~s}^{-1}$, suggesting a moderate to high inclination of the system. A high orbital inclination of HS 1857 was confirmed by the detection of eclipses in the light curves of the system (Fig. 7).

\subsection{Long term variability}

Throughout our photometric observing runs, HS 1857 was found to vary over a relatively large range between $17.2-13.9$ mag in average brightness, suggesting a frequent outburst activity (Fig. 7). Combined with the long orbital period (see below), it appears likely that HS 1857 is a ZCam-type dwarf nova. The INT spectra obtained in April 2003 showed the system with a broad absorption trough around $\mathrm{H} \beta$, with a weak $(E W \sim 3.5 \AA)$ single-peaked emission core, typical of dwarf novae during outburst (Hessman et al. 1984). As we did not obtain a spectrophotometric flux standard on that occasion, and have no simultaneous photometric data, the magnitude of that outburst could not be determined. An additional outburst spectrum was obtained in the ultraviolet using HST/STIS on August 17, 2003, showing a range of low and high ionisation lines of $\mathrm{C}, \mathrm{N}, \mathrm{Si}$, and $\mathrm{Al}$ in absorption, as well as a P-Cygni profile in $\mathrm{C}$ IV $\lambda 1550$ (Fig. 12). We derived an $R$-band equivalent magnitude of 14.1 from the STIS acquisition image taken before the ultraviolet spectroscopy (see Araujo-Betancor et al. 2005b, for details on the processing of STIS acquisition images), and ground-based photometry obtained at Kryoneri a few hours after the STIS observations found HS 1857 at $V=13.9$. The STIS spectrum resembles qualitatively the ultraviolet spectrum of Z Cam obtained during an outburst (Knigge et al. 1997). The P-Cygni profile provides evidence for the presence of a wind outflow during the outburst.

\subsection{Eclipse ephemeris}

We obtained light curves of HS 1857 throughout the period 2002 to 2004 and covered nine eclipses. We measured the times of the eclipse minima, and determined the cycle count by fitting $\left(\phi_{0}^{\mathrm{fit}}-\right.$ $\left.\phi_{0}^{\text {observed }}\right)^{-2}$, leaving the period as a free parameter (Fig. 13). The following ephemeris was derived as:

$\phi_{0}=$ HJD 2452 368.53243(98) $+0.189109(1) \times E$

where $\phi_{0}$ is defined as the phase of mid-eclipse. The errors (given in brackets) of the zero phase and period were determined 


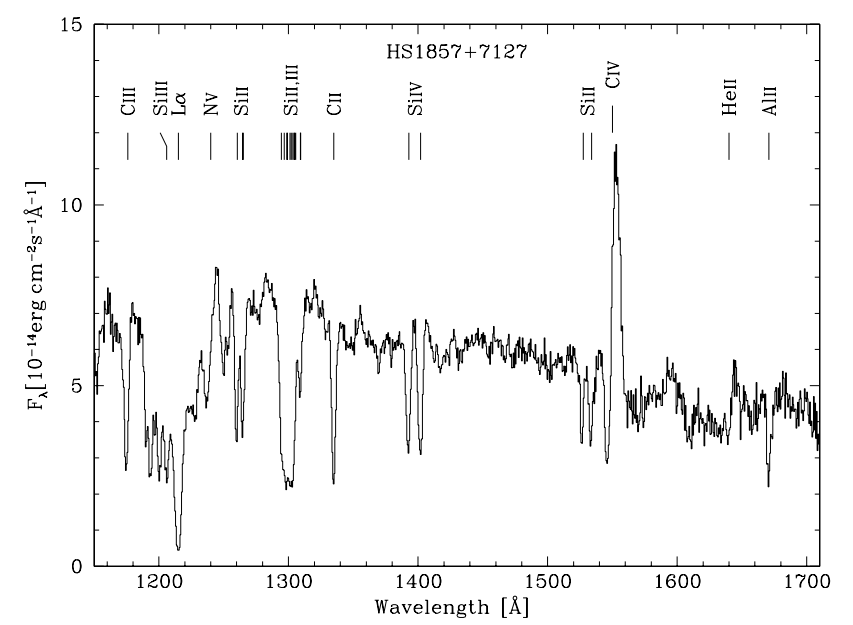

Fig. 12. The HST/STIS spectrum of HS 1857 taken on August 17, 2003 during an outburst.

Table 6. The times of eclipse minima of HS 1857 obtained during the 2002 to 2004 runs.

\begin{tabular}{cc}
\hline \hline Date & Eclipse minima (HJD) \\
\hline 2002 Apr. 03 & 2452368.53446 \\
2002 Apr. 22 & 2452387.44260 \\
2002 Sep. 16 & 2452534.38100 \\
2002 Oct. 24 & $2452572.39500,2452572.57893$ \\
2003 Apr. 22 & $2452752.42275,2452752.61229$ \\
2004 May 03 & 2453128.56222 \\
2004 May 04 & 2453129.88816 \\
\hline
\end{tabular}

from a least-squares fit to the observed eclipse times versus the cycle count number. We conclude that the orbital period of HS 1857 is $P_{\text {orb }}=272.317(1)$ min.

The overall shape of the light curves and that of the eclipse profiles show a large degree of variability (Fig. 7). On April 22, 2002, the light curve shows an orbital modulation with a bright hump preceding the eclipse, typically observed in quiescent eclipsing dwarf novae (e.g. Zhang \& Robinson 1987), produced by the bright spot. A shallow ( $\sim 0.4 \mathrm{mag}$ ) eclipse is recorded, implying a partial eclipse of the accretion disc in the system. On September 16, 2002, the system was apparently caught on the rise to an outburst, with the eclipse depth reduced to $\sim 0.2 \mathrm{mag}$. During several intermediate and bright states the signature of the bright spot disappeared, and was replaced by a broad orbital modulation with maximum light near phase 0.5 , superimposed by short time scale flickering (Fig. 7 , bottom four panels). On May 3, 2004, a narrow $\operatorname{dip}(\Delta \phi \simeq 0.05)$ centred at $\phi \simeq 0.8$ precedes the eclipse during both observed cycles. A similar feature, though of lower depth, has been observed on April 22, 2003.

\subsection{Radial velocities}

As for HS 1016 and HS 1340, we measured the $\mathrm{H} \alpha$ radial velocities of HS 1857 using the double-Gaussian method with a separation of $1500 \mathrm{~km} \mathrm{~s}^{-1}$ and an $F W H M$ of $400 \mathrm{~km} \mathrm{~s}^{-1}$. The Scargle periodogram computed from these data contains a set of narrow peaks at $3.3 \mathrm{~d}^{-1}, 4.3 \mathrm{~d}^{-1}$, and $5.3 \mathrm{~d}^{-1}$, consistent with the photometric frequency, $f \simeq 5.29 \mathrm{~d}^{-1}$, computed from the eclipse ephemeris in the previous section, and its one-day aliases (Fig. 9, third panel). A periodogram calculated from a faked data set assuming the photometric frequency of $5.28796 \mathrm{~d}^{-1}$, is shown in

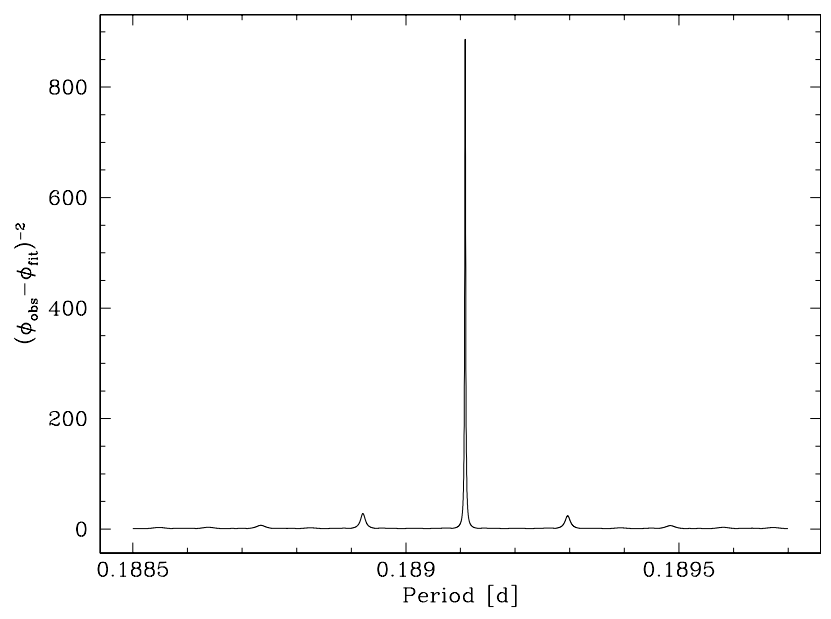

Fig. 13. The periodogram of HS 1857 computed from nine eclipses obtained during the 2002 to 2004 runs.

the insert in Fig. 9 (third panel). We folded the $\mathrm{H} \alpha$ radial velocity curve using the eclipse ephemeris given in Eq. (1), resulting in a quasi-sinusoidal modulation with an amplitude of $128.0 \pm 9.6 \mathrm{~km} \mathrm{~s}^{-1}$ and $\gamma=-17.9 \pm 7.4 \mathrm{~km} \mathrm{~s}^{-1}$, as determined from a sine-fit (Table 5). The red-to-blue crossing of the $\mathrm{H} \alpha$ radial velocities occurs at the photometric phase $\sim 0.1$ (Fig. 10, third panel). Such a shift is not too much of a surprise, as our radial velocity measurements were extracted from spectra sampling different brightness (outburst) states of HS 1857, covering less than one orbital cycle in all cases, and are not expected to represent a uniform and symmetrical emission from the accretion disc.

\section{HS 2214+2845}

The CAFOS average spectrum of HS 2214 (Fig. 2) is characterised by a fairly red continuum superimposed by strong Balmer emission lines and weaker emission lines of $\mathrm{He}$ I, He II, and Fe II. The Balmer line profiles are double-peaked with a peak-to-peak separation of $\sim 800-1000 \mathrm{~km} \mathrm{~s}^{-1}$, suggesting an origin in an accretion disc (Horne \& Marsh 1986). TiO absorption bands are present in the red part of the spectrum, revealing the late-type secondary star.

During our spectroscopic and photometric follow-up studies, HS 2214 was consistently found at $16.5-15.5 \mathrm{mag}$, with nightto-night variations of the mean magnitude of $0.2-0.4 \mathrm{mag}$. The dwarf nova nature of HS 2214 was confirmed through the visual monitoring by one of us (PS), which led to the detection of outbursts on December 10, 2004, and on May 2, July 14, September 22, 2005, and April 23, 2006. The mean cycle length appears to be $\sim 71 \mathrm{~d}$, and the maximum brightness recorded during outburst was $V \simeq 12.3$.

\subsection{The spectral type of the secondary and distance}

Overall, the spectrum of HS 2214 resembles that of the U Gem-type dwarf novae, e.g. CZ Ori (Ringwald et al. 1994), PG 0935+075 (Thorstensen \& Taylor 2001), and U Gem itself (Wade 1979; Stauffer et al. 1979), which have secondary stars with spectral types in the range M2-M4, and orbital periods in the range 255-315 min.

In order to determine the spectral type of the secondary star in HS 2214, we used a library of spectral templates created from Sloan Digital Sky Survey data, covering spectral types 
M0-M9. For each spectral type, we varied the flux contribution of the M-dwarf template until the molecular absorption bands cancelled out as much as possible in the difference spectrum of HS 2214 minus template. The best match in the relative strength of the $\mathrm{TiO}$ absorption bands is achieved for a spectral type M2.5 \pm 0.5 , contributing $25 \%$ of the observed $V$-band flux of HS 2214 (Fig. 14). The extrapolated $J H K_{\mathrm{s}}$ spectrum of the secondary star $^{2}$ agrees fairly well with the 2MASS $J H K_{\mathrm{s}}$ magnitudes of HS 2214 (14.5, 13.9, and 13.5, respectively), suggesting that the accretion disc contributes only a small amount to the infrared flux.

Using Beuermann \& Weichhold's (1999) calibration of the surface brightness in the 7165/7500 $\AA \mathrm{TiO}$ band, and assuming a radius of $R_{2}=(3.0 \pm 0.3) \times 10^{10} \mathrm{~cm}$, based on the orbital period determined below and various radius-orbital period relations (e.g. Warner 1995; Beuermann \& Weichhold 1999), we estimate the distance of HS 2214 to be $d=390 \pm 40 \mathrm{pc}$, where the error is dominated by the uncertainty of the secondary's radius.

\subsection{The orbital period}

We first measured the radial velocity variation of $\mathrm{H} \alpha$ in the INT spectra and in the CAFOS spectra taken with the $R-100$ grism, as well as that of $\mathrm{H} \beta$ in the $B-100$ CAFOS spectra by using the double Gaussian method of Schneider \& Young (1980). The Scargle periodogram calculated from these measurements contained a peak near $\sim 5.5 \mathrm{~d}^{-1}$, but was overall of poor quality. In a second attempt, we determined the $\mathrm{H} \alpha$ and $\mathrm{H} \beta$ radial velocities by means of the $V / R$ ratios, calculated from having equal fluxes in the blue and red line wing, fixing the width of the line to $\sim 2500 \mathrm{~km} \mathrm{~s}^{-1}$ in order to avoid contamination by the $\mathrm{He}$ I $\lambda 6678$ line adjacent to $\mathrm{H} \alpha$. The Scargle periodogram calculated from these sets of radial velocities contains the strongest signal at $6.6 \mathrm{~d}^{-1}$ and an $1 \mathrm{~d}^{-1}$ alias of similar strength at $5.6 \mathrm{~d}^{-1}$ (see Fig. 9, bottom panel). Based on the spectroscopy alone, an unambiguous period determination is not possible.

A crucial clue in determining the orbital period of HS 2214 came from the analysis of the two longest photometric time series obtained at the Braeside Observatory in September 2000 (Fig. 6). These light curves display a double-humped structure with a period of $\sim 4 \mathrm{~h}$, superimposed by relatively low-amplitude flickering. The analysis-of-variance periodogram (AOV, Schwarzenberg-Czerny 1989) calculated from these two light curves contains two clusters of signals in the range of $4-7 \mathrm{~d}^{-1}$ and $10-13 \mathrm{~d}^{-1}$, respectively (see Fig. 15). The strongest peaks in the first cluster are found at $\simeq 5.58 \mathrm{~d}^{-1}$ and $\simeq 5.92 \mathrm{~d}^{-1}$ and at $\simeq 11.14 \mathrm{~d}^{-1}$ and $\simeq 11.48 \mathrm{~d}^{-1}$ in the second cluster. Based on the fact that two of the frequencies are commensurate, we identify $f_{1}=5.58 \mathrm{~d}^{-1}$ and $f_{2}=11.14 \mathrm{~d}^{-1}$ as the correct frequencies, with $f_{1}$ being the fundamental and $f_{2}$ its harmonic. The periodogram of a faked data set computed from a sine wave with a frequency of $5.58 \mathrm{~d}^{-1}$, evaluated at the times of the observations and offset by the randomised observational errors reproduces the alias structure observed in the periodogram of the data over the range $4-7 \mathrm{~d}^{-1}$ very well (Fig. 15, top panel). A two-frequency sine fit with $f_{2}=2 \times f_{1}$ to the data results in $f_{1}=5.581(12) \mathrm{d}^{-1}$. The Braeside photometry folded over that frequency displays a double-hump structure (Fig. 16, two bottom panels). We identify $f_{1}$ as the orbital frequency of the system, hence, $P_{\text {orb }}=$ 258.02(56) min based on the following arguments. (a) The fundamental frequency detected in the photometry coincides with

${ }^{2}$ Using LHS399 from Sandy Leggett's library of M-dwarf spectra, http://ftp.jach.hawaii . edu/ukirt/skl/dM. spectra/

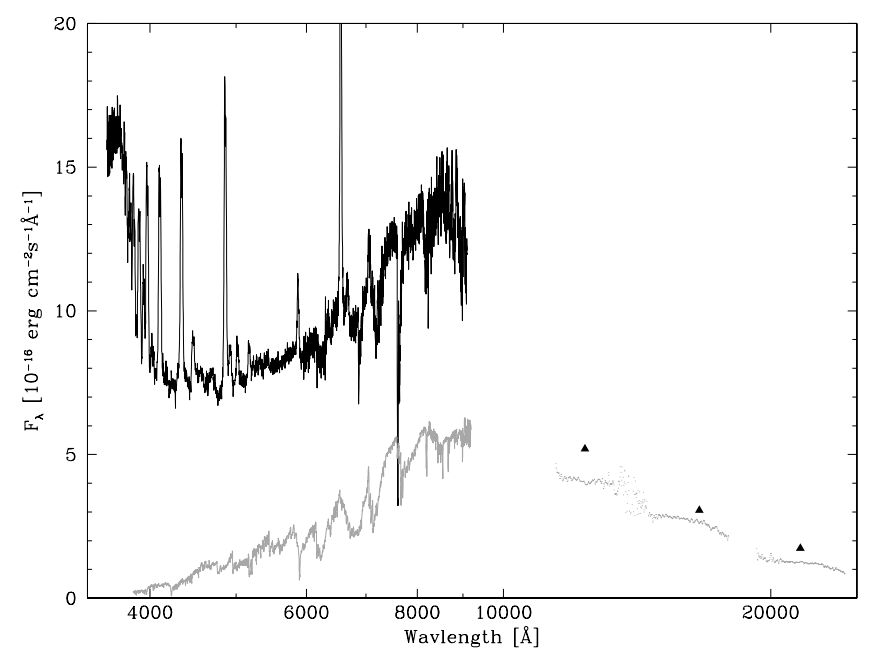

Fig. 14. The average CAFOS spectrum of HS 2214 (black line) along with the best-matching M-dwarf template of spectral type M 2.5, scaled to fit the strength of the molecular bands in HS 2214. The 2MASS $J H K_{\mathrm{s}}$ infrared fluxes of HS 2214 are represented by filled triangles; the $J H K$ spectrum of the M-dwarf template is shown as gray dots.

that of the second-strongest peak in the periodogram determined from the $\mathrm{H} \alpha$ and $\mathrm{H} \beta V / R$-ratio radial velocity measurements (Fig. 9, bottom panel). (b) Double-humped orbital light curves are observed in a large number of short-period dwarf novae, e.g. WX Cet (Rogoziecki \& Schwarzenberg-Czerny 2001), WZ Sge (Patterson 1998), RZ Leo, BC UMa, MM Hya, AO Oct, HV Vir (Patterson et al. 2003), HS 2331+3905 (Araujo-Betancor et al. 2005a), and HS 2219+1824 (Rodríguez-Gil et al. 2005a); the origin of those double-humps is not really understood, but most likely associated with the accretion disc/bright spot. In longperiod dwarf novae, double-humped light curves are observed in the red part of the spectrum caused by ellipsoidal modulation of the secondary star, e.g. U Gem (Berriman et al. 1983) or IP Peg (Szkody \& Mateo 1986; Martin et al. 1987). In both cases, a strong and sometimes dominant, signal at the harmonic of the orbital period is seen in the periodogram calculated from their light curves.

Figure 10 (bottom panel) shows the radial velocity data folded over the photometric orbital period (258.02 min), along with a sine-fit (Table 5). The radial velocities are shown again in Fig. 16 together with the Braeside photometry, all folded using the photometric period but the spectroscopic zeropoint (Table 5). The photometric minima occur near orbital phase zero (inferior conjunction of the secondary) and 0.5 , consistent with what is expected for ellipsoidal modulation. Very similar phasing is observed also for the double-humps in short-period systems, e.g. WZ Sge (Patterson 1980) shows maximum brightness close to phases 0.25 and 0.75 . However, given the strong contribution of the secondary star to optical flux of HS 2214 (Fig. 14), and the fact that the filterless Braeside photometry is rather sensitive in the red, we believe that the origin of the double-hump pattern seen in HS 2214 is indeed ellipsoidal modulation.

The binary parameters of HS 2214 could be improved in a future study by a measurement of the radial velocity of the secondary star, e.g. using the Na doublet in the $I$ band, and a determination of the orbital inclination from modelling the ellipsoidal modulation. 


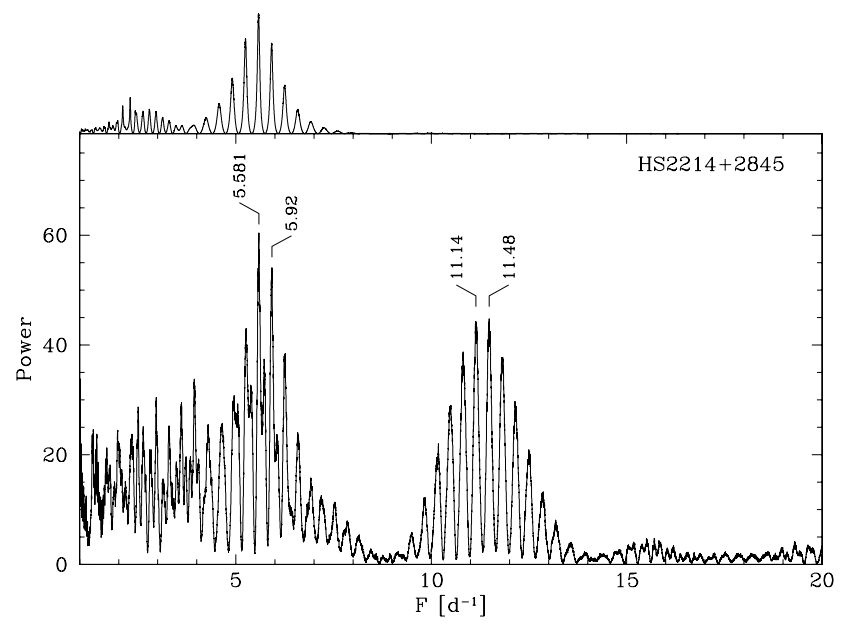

Fig. 15. Main panel: the analysis-of-variance (AOV) periodogram of HS 2214 calculated from the two longest light curves obtained at the Braeside Observatory, which show a double-humped pattern. Top panel: the AOV periodogram created from a sine wave with the orbital period of $258.02 \mathrm{~min}$.

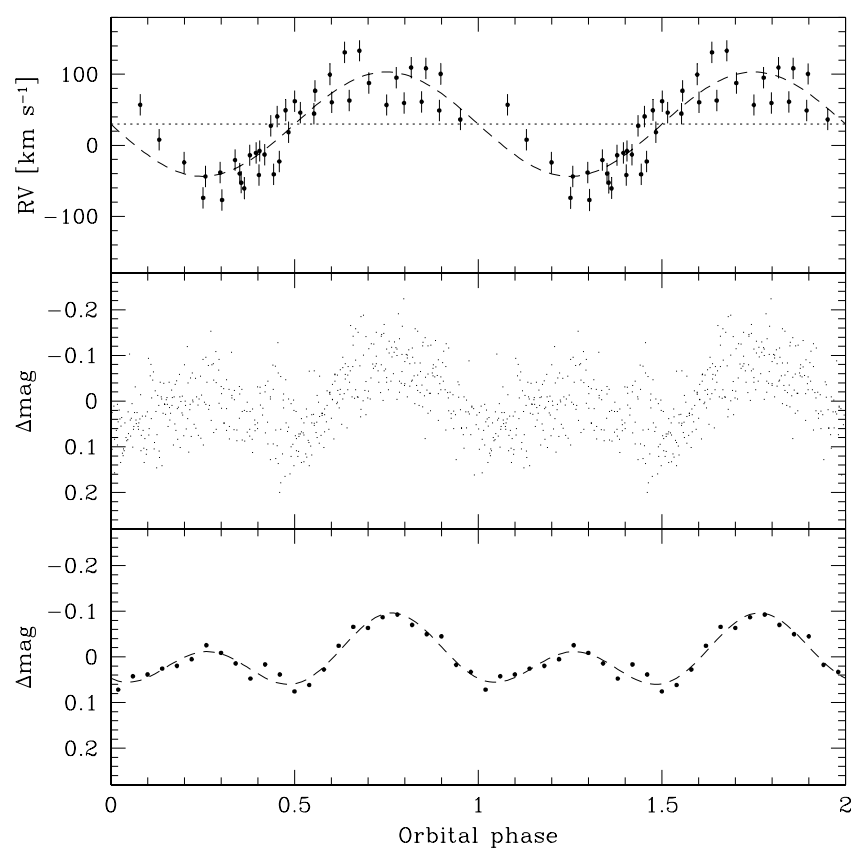

Fig. 16. The spectroscopic and photometric data of HS 2214 are folded on the photometric period of $258.02 \mathrm{~min}$ using the spectroscopic zeropoint $T_{0}=$ HJD 2451812.3309 defined by the red-to-blue crossing of the $\mathrm{H} \alpha$ and $\mathrm{H} \beta$ radial velocities. In that phase convention, the inferior conjunction of the secondary star is expected at orbital phase zero. Top panel: the radial velocity variation of the $\mathrm{H} \alpha$ and $\mathrm{H} \beta$ emission lines, as already shown in Fig. 10. A whole cycle has been repeated for clarity. Middle panel: the photometric data obtained from the Braeside Observatory. Bottom panel: the photometric data binned into 25 phase slots, along with a two-frequency sine fit (dashed line).

\section{The orbital period distribution of dwarf novae}

Because of their outbursts, the vast majority of all currently known dwarf novae have been discovered by variability surveys, either through professional sky patrols, or through the concentrated efforts of a large number of amateur astronomers (Gänsicke 2005). Considering the irregular temporal sampling of such observations, the population of known dwarf novae is

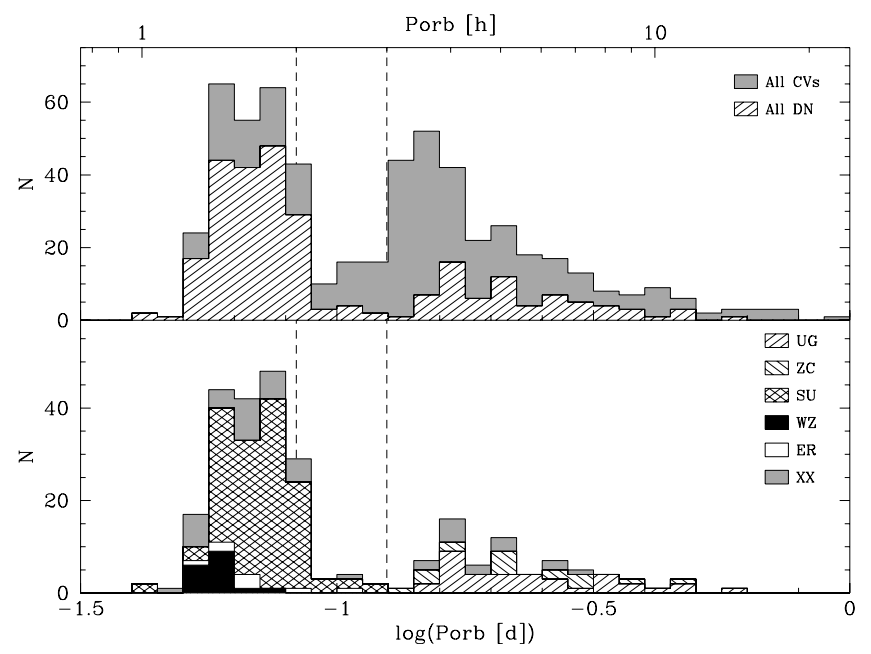

Fig. 17. Top panel: the orbital period distribution of known CVs and dwarf novae from Ritter \& Kolb (2003, 7th edition, rev. 7.5, July 2005) are shown in gray and shade, respectively. Bottom panel: the period distribution of known dwarf novae according to their subtype, U Gem (UG), Z Cam (ZC), SU UMa (SU), WZ Sge (WZ), ER UMa (ER), and unclassified subtype (XX). The dashed lines are the conventional 2-3 h period gap.

likely to be biased towards systems which have frequent and/or large amplitude outbursts.

\subsection{The orbital period distribution of all known dwarf novae}

Inspecting the Ritter \& Kolb catalogue (2003, edition 7.5 of July 1,2005 ) within the orbital period range of $\sim 1 \mathrm{~h}$ to $\sim 1 \mathrm{~d}$ and removing AMCVn systems, it is found that nearly half of all known CVs (262 systems out of 572, or 46\%) are dwarf novae of which $166(63 \%)$ have $P_{\text {orb }}<2 \mathrm{~h}, 26(10 \%)$ are found in the $2-3 \mathrm{~h}$ orbital period gap, and $70(27 \%)$ have long periods, $P_{\text {orb }}>3 \mathrm{~h}$. The conventional definition of the period gap as being the range $2-3 \mathrm{~h}$ is somewhat arbitrary, and these numbers vary slightly if a different definition is used, but without changing the overall picture. Figure 17 (top panel) shows the orbital period distribution of all known CVs and dwarf novae with periods between $\sim 1 \mathrm{~h}$ and $\sim 1 \mathrm{~d}$. Whereas the total population of all $\mathrm{CV}$ s features the well-known period gap, i.e. the relatively small number of $\mathrm{CVs}$ with periods $2 \mathrm{~h}<P_{\text {orb }}<3 \mathrm{~h}$, the number of dwarf nova reaches a minimum in the range $3-4 \mathrm{~h}$. In fact, the number of dwarf nova with $3 \mathrm{~h}<P_{\text {orb }}<4 \mathrm{~h}$ is a half $(15$, or $6 \%$ of all dwarf novae) of that in the "standard" $2-3 \mathrm{~h}$ period gap (26, or $10 \%)$. The dearth of known dwarf novae in the 3-4 h period range was pointed out by Shafter et al. (1986) and Shafter (1992), who compared the observed dwarf nova period distribution with those constructed from various magnetic braking models, and concluded that none of the standard magnetic braking models can satisfactorily explain the lack of observed dwarf novae in the $3-4 \mathrm{~h}$ period range.

The bottom panel of Fig. 17 displays all known dwarf novae according to their subtypes which are $159(61 \%)$ SU UMa, 37 (14\%) U Gem, 18 (7\%) Z Cam, and 48 (18\%) unclassified subtypes (XX). For completeness, we note that the SUUMa class includes 8 ER UMa stars (which have very short superoutburst cycles) and 19 WZ Sge stars (which have extremely long outburst cycles). All confirmed U Gem and Z Cam stars lie above the period gap, in fact all but one Z Cam star (BX Pup) 
have $P_{\text {orb }}>3.5 \mathrm{~h}^{3}$. It is clearly seen that the majority $(85 \%)$ of SU UMa lie below the period gap and only a small fraction $(15 \%)$ inhabits the $2-3 \mathrm{~h}$ period range ${ }^{4}$.

The orbital period distribution of short-period dwarf novae in Fig. 17 (the majority of all CVs in this period range) differs markedly from the predictions made by the standard CV evolution theory (e.g. Kolb 1993; Kolb \& Baraffe 1999; Howell et al. 2001): the minimum period is close to $\sim 77 \mathrm{~min}$, contrasting with the predicted minimum period of $\sim 65$ min (Paczyński \& Sienkiewicz 1983), and the distribution of systems is nearly flat in $P_{\text {orb }}$, whereas the theory predicts a substantial accumulation of systems at the minimum period. Several modifications of the CV evolution theory have been suggested to resolve this discrepancy, however, none with indisputable success (King et al. 2002; Renvoizé et al. 2002; Barker \& Kolb 2003).

\subsection{The orbital period distribution of dwarf novae in the HQS}

Another possible explanation for the lack of a spike in the orbital period distribution of CVs near the minimum period is that systems close to the minimum period, especially those evolving back to longer periods, have not yet been discovered due to observational selection effects. As most CVs below the period gap are dwarf novae, the most obvious bias suppressing the period spike is to assume that dwarf novae close to the minimum period have very rare outbursts. In fact, a number of dwarf novae near the minimum period have very long outburst intervals, e.g. WZ Sge $\left(P_{\text {orb }}=81.6 \mathrm{~min}\right.$, Patterson et al. 2002) erupts every 20-30 years; GW Lib $\left(P_{\text {orb }}=76.8 \mathrm{~min}\right.$, Thorstensen et al. 2002) has been seen in outburst only once in 1983 . It can not be excluded that these systems represent "the tip of the iceberg" of a dwarf nova population with even longer outburst periods. Assuming that rarely outbursting dwarf novae do exist in a significant number, and that they spectroscopically resemble the known objects, such as WZ Sge or GW Lib, our search for $\mathrm{CVs}$ in the HQS should be able to identify them (Gänsicke et al. 2002).

We have so far obtained orbital periods for 41 new CVs found in the HQS; their period distribution is shown in Fig. 18 (top panel). The first thing to notice is that the majority of the new CVs identified in the HQS are found above the period gap, with a large number of systems in the period range $3-4 \mathrm{~h}$ (for a discussion of the properties of CVs in this period range, see Aungwerojwit et al. 2005). As for the overall CV population, a dearth of systems is observed in the $2-3 \mathrm{~h}$ period range (Fig. 18, top panel), with the gap being wider for dwarf novae (Fig. 18, bottom panel).

To date $14(26 \%)$ out of 53 new CVs discovered in the HQS have been classified as dwarf novae, including the five systems, HS 0417, HS 1016, HS 1340, HS 1857, and HS 2214, presented in this paper (Table 7). The fraction of long-period $\left(P_{\text {orb }}>3 \mathrm{~h}\right)$ systems is larger in the HQS sample $(43 \%)$ than in the total population of known dwarf novae (27\%, see Sect. 8.1).

${ }^{3}$ Ritter \& Kolb (2003) list five U Gem-type dwarf novae with $P_{\text {orb }}<$ $3 \mathrm{~h}$ : CC Cnc is a SU UMa-type dwarf nova (Kato \& Nogami 1997), and we included $587 \mathrm{Lyr}$, CF Gru, V544 Her, and FS Aur as dwarf novae with no subtype $(\mathrm{XX})$ due to the lack of clear observational evidence for a specific subtype.

${ }^{4}$ A note of caution among the WZSge stars, which mostly have ultrashort-periods, concerns UZ Boo. Ritter \& Kolb (2003) list a period of $\sim 3 \mathrm{~h}$ based on quiescent photometry, which is almost certainly wrong. Intensive time-series of the 2003 outburst of UZ Boo revealed a superhump period of $89.3 \mathrm{~min}$ (Kato, vsnet-campaign-dn 4064), and we use here this value as an estimate of the orbital period.

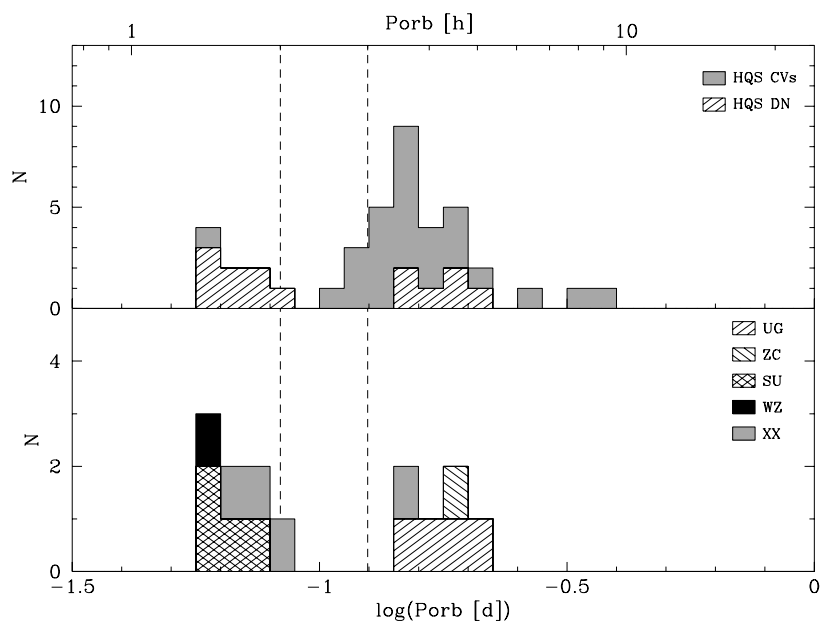

Fig. 18. Top panel: the orbital period distribution of 41 new CVs and 14 dwarf novae identified in HQS are shown in gray and shade, respectively. Bottom panel: the period distribution of HQS dwarf novae according to their subtype, U Gem (UG), Z Cam (ZC), SU UMa (SU), WZ Sge (WZ), and unclassified subtype (XX). The dashed lines are the conventional $2-3 \mathrm{~h}$ period gap.

Table 7. Dwarf novae discovered in the HQS with their subtype, U Gem (UG), SU UMa (SU), ZCam (ZC), and unclassified (XX). Uncertain classifications are marked by a colon.

\begin{tabular}{llccc}
\hline \hline HQS ID & Other name & $P_{\text {orb }}(\min )$ & Type & References \\
\hline HS 2331+3905 & & 81.1 & WZ: & 1 \\
HS 1449+6415 & KV Dra & 84.9 & SU & 2,3 \\
HS 2219+1824 & & 86.3 & SU & 4 \\
HS 1340+1524 & & 92.7 & XX & 5 \\
HS 1017+5319 & KS UMa & 97.9 & SU & 2,6 \\
HS 0417+7445 & & $105.1 / 109.9$ & SU & 5 \\
HS 1016+3412 & \multirow{2}{*}{ GZ Cnc } & 114.3 & XX & 5 \\
HS 0913+0913 & 127.1 & XX & $2,8,9$ \\
HS 0941+0411 & RX J0944.5+0357 & 215.0 & XX & 2,10 \\
HS 0552+6753 & LU Cam & 216.6 & UG & 2,7 \\
HS 0907+1902 & GY Cnc & 252.6 & UG & 2,11 \\
HS 2214+2845 & & 258.0 & UG & 5 \\
HS 1857+7127 & \multirow{2}{*}{ EX Dra } & 272.3 & ZC: & 5 \\
HS 1804+6753 & EX & 302.3 & UG & $12,13,14$ \\
\hline
\end{tabular}

References: (1) Araujo-Betancor et al. (2005a); (2) Jiang et al. (2000); (3) Nogami et al. (2000); (4) Rodríguez-Gil et al. (2005a); (5) this work; (6) Patterson et al. (2003); (7) Thorstensen priv. com., \& vsnetcampaign-dn 2681; (8) Kato et al. (2002); (9) Tappert \& Bianchini (2003); (10) Mennickent et al. (2002); (11) Gänsicke et al. (2000); (12) Fiedler et al. (1997); (13) Billington et al. (1996); (14) Shafter \& Holland (2003).

The total number of new HQS dwarf novae is relatively small, and subject to corresponding statistical uncertainties. However, the tilt towards long-period dwarf novae among the new HQS CVs is likely to be underestimated, as a significant number of long-period HQS CVs have still uncertain CV subtypes, and several of them could turn out to be additional a ZCam-type dwarf nova (Aungwerojwit et al. 2005 plus additional unpublished data). Optical monitoring of the long-term variability of these systems will be necessary to unambiguously determine their CV type. Overall, the dwarf novae identified within the HQS fulfill the above expectations of being "low-activity" systems, i.e. dwarf novae that have either infrequent outbursts (e.g. KV Dra, HS 0941+0411, HS 2219+1824) or low-amplitude 
outbursts (e.g. EX Dra). We found only one system that resembles the WZSge stars with their very long outburst intervals found near the minimum period that is HS 2331+3905 (AraujoBetancor et al. 2005a) which has a period of $81.1 \mathrm{~min}$, and no outburst has been detected so far.

Thus, our search for CVs in the HQS has been unsuccessful in identifying the predicted large number of short-period CVs, despite having a very high efficiency in picking up systems that resemble the typical known short-period dwarf novae.

\section{Constraints on the space density of CVs}

$\mathrm{CV}$ population models result in space densities in the range $2 \times 10^{-5} \mathrm{pc}^{-3}$ to $2 \times 10^{-4} \mathrm{pc}^{-3}$ (de Kool 1992; Politano 1996), whereas the space density determined from observations is $(0.5-1) \times 10^{-5} \mathrm{pc}^{-3}$ (Patterson 1984; Ringwald 1996; Patterson 1998). It appears therefore that we currently know about an order of magnitude less CVs than predicted by the models. Also the observed ratio of short to long orbital period systems is in strong disagreement with the predictions of the theory. Patterson $(1984,1998)$ estimated that the fraction of short-period CVs per volume is $75-80 \%$, which has to be compared to $99 \%$ in the population studies (Kolb 1993; Howell et al. 1997).

Because of the large differences in mass transfer rates, and, hence, absolute magnitudes, of long $\left(P_{\text {orb }}>3 \mathrm{~h}\right)$ and short $\left(P_{\text {orb }}<3 \mathrm{~h}\right)$ period CVs, magnitude limited samples appear at a first glance utterly inappropriate for the discussion of $\mathrm{CV}$ space densities. However, taking the theoretical models at face value, the space density of long period CVs is entirely negligible compared to that of short period CVs (Kolb 1993; Howell et al. 1997), and hence a discussion of the total CV space density can be carried out on the basis of short-period systems alone. In the following, we assess the expected numbers of systems in the HQS separately for short-period CVs that are still evolving towards the minimum period (pre-bounce), and those that already reached the minimum period, and evolve back to longer periods (post-bounce). For both cases, we assumed (1) a space density of $5 \times 10^{-5} \mathrm{pc}^{-3}$ as an intermediate value between the predictions of de Kool (1992) and Politano (1996), (2) that $70 \%$ of all CVs are post-bounce systems, and $30 \%$ are pre-bounce systems (Kolb 1993; Howell et al. 1997) (ignoring, as stated above, the small number of long-period CVs), (3) a scale height of $150 \mathrm{pc}$ (e.g. Patterson 1984), and (4) that the luminosity of short-period CVs is dominated by the accretion-heated white dwarf.

\subsection{Pre-bounce CVs expected in the HQS}

$\mathrm{CV}$ evolution models predict a typical accretion rate of $\dot{M} \simeq$ $5 \times 10^{-11} M_{\odot} \mathrm{yr}^{-1}$ for pre-bounce systems, with a relatively small spread (Kolb 1993; Howell et al. 1997). For this accretion rate, Townsley \& Bildsten's (2003) calculation of white dwarf accretion heating predicts an effective temperature of $T_{\mathrm{wd}} \simeq 12000 \mathrm{~K}$. If the luminosity is dominated by a white dwarf of this temperature, the HQS with an average magnitude limit of $B \simeq 18$ would detect pre-bounce CVs out to a distance of $175 \mathrm{pc}$ (using the absolute magnitudes of white dwarfs by Bergeron et al. 1995 ), i.e. over a bit more than one scale height. Within a sphere of radius $175 \mathrm{pc}$ around the Earth, one would expect $\simeq 144 \mathrm{CVs}$ (taking into account the exponential drop-off of systems perpendicular to the plane), of which $\simeq 36$ would be within the sky area sampled by the HQS. This is a conservative lower limit, as any additional luminosity from the accretion disc and/or bright spot as well as a hotter white dwarf temperature would increase the volume sampled by the HQS, and therefore increase the number of pre-bounce CVs within the survey.

\subsection{Post-bounce CVs expected in the HQS}

Once past the minimum period, the accretion rate of CVs substantially drops as a function of time, and we assume here a value of $\dot{M}=10^{-11} M_{\odot} \mathrm{yr}^{-1}$, corresponding to an intermediate CV age of $\sim 5$ Gyr (Kolb \& Baraffe 1999), and a white dwarf temperature of $T_{\mathrm{wd}} \simeq 7000 \mathrm{~K}$ (Townsley \& Bildsten 2003). This lower white dwarf temperature reduces the detection limit of the HQS to only $\simeq 65 \mathrm{pc}$. The total number of post-bounce $\mathrm{CVs}$ within a sphere of radius $65 \mathrm{pc}$ around the Earth is hence expected to be 40 , of which 10 within the HQS area.

\subsection{Short-period CVs in the HQS: most likely pre-bounce only}

The immediate question is what type are the short-period HQS CVs: pre- or post-bounce? As mentioned in Sect. 8.2, only 12 short-period $\left(P_{\text {orb }}<3 \mathrm{~h}\right)$ systems have been found among the 41 new HQS CVs for which we have adequate follow-up data. The new short-period CVs comprise 8 dwarf novae (Table 7), two polars (Reimers et al. 1999; Jiang et al. 2000; Schwarz et al. 2001; Tovmassian et al. 2001; Thorstensen \& Fenton 2002; Gänsicke et al. 2004b), one intermediate polar (Rodríguez-Gil et al. 2004a; Patterson et al. 2004), and one system with uncertain classification (Gänsicke et al. 2004a). The white dwarf has been detected in the spectra of HS 2237+8154 (Gänsicke et al. 2004a), HS 2331+3905 (Araujo-Betancor et al. 2005a), HS 2219+1824 (Rodríguez-Gil et al. 2005a), and HS 1552+2730 (Gänsicke et al. 2004b), with temperatures of $\simeq 10500 \mathrm{~K}$, $\simeq 11500 \mathrm{~K}, \simeq 15000 \mathrm{~K}$, and $\simeq 20000 \mathrm{~K}$, respectively. These four systems are very likely to have the lowest mass transfer rates among the 12 new short-period CVs; the optical spectra of the other eight are characterised by strong Balmer and He emission lines and the associated continuum which outshines the white dwarf, typical of higher accretion rates. While there are still about a dozen HQS CVs with no accurate orbital period determination, the data already at hand makes it very unlikely that more than 2 or 3 of those systems will turn out to have periods $<3 \mathrm{~h}$.

For a complete assessment of the short-period content of the HQS, one has obviously to include in the statistics the shortperiod CVs that are contained within the HQS data base, but were already known - subject to the same selection criteria that were applied to identify the twelve new systems. Gänsicke et al. (2002) analysed the properties of the previously known CVs within the HQS data base, and came to the following conclusions. 18 previously known short-period $\left(P_{\text {orb }}<3 \mathrm{~h}\right)$ systems with HQS spectra are correctly (re-)identified as CVs, including 12 dwarf novae, five polars, and one intermediate polar ${ }^{5}$. Gänsicke et al. (2002) also found that only two previously known short-period systems with HQS spectra failed to be identified as CVs; this "hit rate" of $90 \%$ underlines the extreme efficiency of the HQS of finding short period CVs. Five out of those 18 systems have measured white dwarf temperatures, all of them in the range $\simeq 11000-16000 \mathrm{~K}$ (MR Ser, ST LMi, AR UMa, SW UMa, T Leo: Gänsicke et al. 2001; Hamilton \& Sion 2004; Araujo-Betancor et al. 2005b; Gänsicke et al. 2005). The remaining 13 systems all have spectra dominated by strong

\footnotetext{
5 Excluding the double-degenerate helium CVs, which follow a different evolution channel that is not taken into account in the population models of de Kool (1992) and Politano (1996).
} 
Balmer and He emission, suggesting accretion rates too high to detect the white dwarf.

In summary, the HQS contains a total of 30 short-period CVs (12 new identifications plus 18 previously known systems); all of which are consistent with being pre-bounce systems. At face value, this number agrees rather nicely with the 36 expected systems derived above, but one has to bear in mind that this number is an absolute lower limit, as hotter white dwarfs and/or accretion luminosity from the disc and hot spot will increase the volume sampled by the HQS.

While there may still be some shortfall of pre-bounce systems, it is much more worrying that so far no systems with the clear signature of a post-bounce $\mathrm{CV}$ that evolved significantly back to longer periods has been found - neither in the HQS, nor elsewhere. The coldest CV white dwarfs have been found, to our knowledge, in the polar EF Eri $\left(T_{\mathrm{wd}} \simeq 9500 \mathrm{~K}\right.$, Beuermann et al. $2000)$, and HS $2331+3905\left(T_{\mathrm{wd}} \simeq 10500 \mathrm{~K}\right.$, Araujo-Betancor et al. 2005a), both systems with orbital periods of $\simeq 81 \mathrm{~min}-$ which may hence be either pre- or post-bounce systems.

A final note concerns the number of WZSge stars, i.e. short-period dwarf novae with extremely long outburst intervals. Given the strong Balmer lines in the known WZ Sge stars e.g. WZ Sge itself (Gilliland et al. 1986), BW Scl (Abbott et al. 1997), GD 552 (Hessman \& Hopp 1990), and GW Lib (Szkody et al. 2000; Thorstensen et al. 2002), we believe that any WZ Sge brighter than $B \simeq 18$ would have easily been identified in the HQS - yet, only a single new WZ Sge system has been discovered, HS 2331+3905 (Araujo-Betancor et al. 2005a).

Thus, we conclude that while our systematic effort in identifying new CVs leads to a space density of pre-bounce short period CVs which agrees with the predictions within an order of magnitude, the bulk of all CVs, which are predicted to have made it past the minimum orbital period, remains unidentified so far.

\section{Conclusions}

We have identified five new dwarf novae as a part of our search for new CVs in the HQS, bringing the total number of HQSdiscovered dwarf novae to 14 . The new systems span orbital periods from $\sim 1.5 \mathrm{~h}$ to nearly $5 \mathrm{~h}$, confirming the trend that dwarf novae spectroscopically selected in the HQS display a larger ratio of long-to-short orbital periods. Overall, dwarf novae represent only about one third of the HQS CVs which are studied sufficiently well, and it is by now clear that the properties of the sample of new HQS CVs do not agree with those of the predicted large population of short-period low-mass-transfer systems. Within the limiting magnitude of $B \simeq 17.5-18.5$, the HQS does however contain a large number of previously known dwarf novae that were identified because of their variability, and almost all of these systems have been recovered as strong CV candidates on the basis of their HQS spectrum (Gänsicke et al. 2002). Based on their spectroscopic and photometric properties it appears that most, if not all short-period CVs in the HQS (newly identified and previously known) are still evolving towards the minimum period. If the large number of post-bounce CVs evolving back to longer periods predicted by population models exists, they must (a) have very long outburst recurrence times, and (b) have $\mathrm{H} \beta$ equivalent widths that are far lower than observed in the currently known typical short-period CVs.

Acknowledgements. A.A. thanks the Royal Thai Government for a studentship. B.T.G. and P.R.G. were supported by a PPARC Advanced Fellowship and a
PDRA grant, respectively. M.A.P.T. is supported by NASA LTSA grant NAG5-10889. R.S. is supported by the Deutsches Zentrum für Luft und Raumfahrt (DLR) GmbH under contract No. FKZ 50 OR 0404. A.S. is supported by the Deutsche Forschungsgemeinschaft through grant Schw536/20-1. The HQS was supported by the Deutsche Forschungsgemeinschaft through grants Re 353/11 and $\operatorname{Re} 353 / 22$. We thank Tanya Urrutia for carrying out a part of the AIP observations. P.S. thanks Robert Mutel (University of Iowa) and his students for taking CCD images with the Rigel telescope. Tom Marsh is acknowledged for developing and sharing his reduction and analysis package MOLLY. This publication makes use of data products from the Two Micron All Sky Survey, which is a joint project of the University of Massachusetts and the Infrared Processing and Analysis Center/California Institute of Technology, funded by the National Aeronautics and Space Administration and the National Science Foundation. Based in part on observations collected at the Centro Astronómico Hispano Alemán (CAHA) at Calar Alto, operated jointly by the Max-Planck Institut für Astronomie and the Instituto de Astrofísica de Andalucía (CSIC); on observations made at the $1.2 \mathrm{~m}$ telescope, located at Kryoneri Korinthias, and owned by the National Observatory of Athens, Greece; on observations made with the IAC80 telescope, operated on the island of Tenerife by the Instituto de Astrofísica de Canarias (IAC) at the Spanish Observatorio del Teide; on observations made with the OGS telescope, operated on the island of Tenerife by the European Space Agency, in the Spanish Observatorio del Teide of the IAC; on observations made with the Isaac Newton Telescope, which is operated on the island of La Palma by the Isaac Newton Group in the Spanish Observatorio del Roque de los Muchachos of the IAC; on observations made at the Wendelstein Observatory, operated by the Universitäts-Sternwarte München; on observations made with the $1.2 \mathrm{~m}$ telescope at the Fred Lawrence Whipple Observatory, a facility of the Smithsonian Institution; and on observations made with the NASA/ESA Hubble Space Telescope, obtained at the Space Telescope Science Institute, which is operated by the Association of Universities for Research in Astronomy, Inc., under NASA contract NAS 5-26555.

\section{References}

Abbott, T. M. C., Fleming, T. A., \& Pasquini, L. 1997, A\&A, 318, 134 Andronov, N., Pinsonneault, M., \& Sills, A. 2003, ApJ, 582, 358

Araujo-Betancor, S., Gänsicke, B. T., Hagen, H.-J., et al. 2005a, A\&A, 430, 629 Araujo-Betancor, S., Gänsicke, B. T., Long, K. S., et al. 2005b, ApJ, 622, 589 Aungwerojwit, A., Gänsicke, B. T., Rodríguez-Gil, P., et al. 2005, A\&A, 443, 995

Barker, J., \& Kolb, U. 2003, MNRAS, 340, 623

Bergeron, P., Wesemael, F., \& Beauchamp, A. 1995, PASP, 107, 1047 Berriman, G., Beattie, D. H., Gatley, I., et al. 1983, MNRAS, 204, 1105 Bertin, E., \& Arnouts, S. 1996, A\&AS, 117, 393

Beuermann, K., \& Weichhold, M. 1999, in Annapolis Workshop on Magnetic Cataclysmic Variables, ed. C. Hellier, \& K. Mukai, ASP Conf. Ser., 157, 283 Beuermann, K., Wheatley, P., Ramsay, G., Euchner, F., \& Gänsicke, B. T. 2000, A\&A, 354, L49

Billington, I., Marsh, T. R., \& Dhillon, V. S. 1996, MNRAS, 278, 673

Cannizzo, J. K. 1993, in Accretion disks in compact stellar objects, ed. J. Wheeler, Advanced Series in Astrophysics and Cosmology No. 9 (Singapore: World Scientific), 6

Cannizzo, J. K., Wheeler, J. C., \& Polidan, R. S. 1986, ApJ, 301, 634 de Kool, M. 1992, A\&A, 261, 188

Fiedler, H., Barwig, H., \& Mantel, K. H. 1997, A\&A, 327, 173

Gänsicke, B. T. 2005, in The Astrophysics of Cataclysmic Variables and Related

Objects, ed. J.-M. Hameury, \& J.-P. Lasota, ASP Conf. Ser., 330, 3

Gänsicke, B. T., Fried, R. E., Hagen, H.-J., et al. 2000, A\&A, 356, L79

Gänsicke, B. T., Schmidt, G. D., Jordan, S., \& Szkody, P. 2001, ApJ, 555, 380

Gänsicke, B. T., Hagen, H. J., \& Engels, D. 2002, in The Physics of Cataclysmic

Variables and Related Objects, ed. B. T. Gänsicke, K. Beuermann, \&

K. Reinsch, ASP Conf. Ser., 261, 190

Gänsicke, B. T., Araujo-Betancor, S., Hagen, H.-J., et al. 2004a, A\&A, 418, 265 Gänsicke, B. T., Jordan, S., Beuermann, K., et al. 2004b, ApJ, 613, L141

Gänsicke, B. T., Szkody, P., Howell, S. B., \& Sion, E. M. 2005, ApJ, 629, 451 Gilliland, R. L., Kemper, E., \& Suntzeff, N. 1986, ApJ, 301, 252

Hagen, H. J., Groote, D., Engels, D., \& Reimers, D. 1995, A\&AS, 111, 195 Hamilton, R. T., \& Sion, E. M. 2004, PASP, 116, 926

Hessman, F. V., \& Hopp, U. 1990, A\&A, 228, 387

Hessman, F. V., Robinson, E. L., Nather, R. E., \& Zhang, E.-H. 1984, ApJ, 286, 747

Horne, K., \& Marsh, T. R. 1986, MNRAS, 218, 761

Howell, S. B., Nelson, L. A., \& Rappaport, S. 2001, ApJ, 550, 897

Howell, S. B., Rappaport, S., \& Politano, M. 1997, MNRAS, 287, 929

Jiang, X. J., Engels, D., Wei, J. Y., Tesch, F., \& Hu, J. Y. 2000, A\&A, 362, 263

Kato, T., \& Nogami, D. 1997, PASJ, 49, 341 
Kato, T., Dubovsky, P. A., Stubbings, R., et al. 2002, A\&A, 396, 929

King, A. R. 1988, QJRAS, 29, 1

King, A. R., Schenker, K., \& Hameury, J. M. 2002, MNRAS, 335, 513

Knigge, C., Long, K. S., Blair, W. P., \& Wade, R. A. 1997, ApJ, 476, 291

Kolb, U. 1993, A\&A, 271, 149

Kolb, U., \& Baraffe, I. 1999, MNRAS, 309, 1034

Martin, J. S., Jones, D. H. P., \& Smith, R. C. 1987, MNRAS, 224, 1031

Mennickent, R. E., Tovmassian, G., Zharikov, S. V., et al. 2002, A\&A, 383, 933

Monet, D. G., Levine, S. E., Canzian, B., et al. 2003, AJ, 125, 984

Nogami, D., Engels, D., Gänsicke, B. T., et al. 2000, A\&A, 364, 701

Osaki, Y. 1996, PASP, 108, 39

Paczyński, B., \& Sienkiewicz, R. 1983, ApJ, 268, 825

Patterson, J. 1980, ApJ, 241, 235

Patterson, J. 1984, ApJS, 54, 443

Patterson, J. 1998, PASP, 110, 1132

Patterson, J., Masi, G., Richmond, M. W., et al. 2002, PASP, 114, 721

Patterson, J., Thorstensen, J. R., Kemp, J., et al. 2003, PASP, 115, 1308

Patterson, J., Thorstensen, J. R., Vanmunster, T., et al. 2004, PASP, 116, 516

Patterson, J., Kemp, J., Harvey, D. A., et al. 2005, PASP, 117, 1204

Politano, M. 1996, ApJ, 465, 338

Rappaport, S., Joss, P. C., \& Verbunt, F. 1983, ApJ, 275, 713

Reimers, D., Hagen, H. J., \& Hopp, U. 1999, A\&A, 343, 157

Renvoizé, V., Baraffe, I., Kolb, U., \& Ritter, H. 2002, A\&A, 389, 485

Ringwald, F. A. 1996, in Cataclysmic Variables and Related Objects, ed.

A. Evans, \& J. H. Wood (Dordrecht: Kluwer), IAU Coll., 158, 89

Ringwald, F. A., Thorstensen, J. R., \& Hamwey, R. M. 1994, MNRAS, 271, 323

Ritter, H., \& Kolb, U. 2003, A\&A, 404, 301

Rodríguez-Gil, P., Gänsicke, B. T., Araujo-Betancor, S., \& Casares, J. 2004a, MNRAS, 349, 367

Rodríguez-Gil, P., Gänsicke, B. T., Barwig, H., Hagen, H.-J., \& Engels, D. 2004b, A\&A, 424, 647
Rodríguez-Gil, P., Gänsicke, B. T., Hagen, H.-J., et al. 2005a, A\&A, 431, 269 Rodríguez-Gil, P., Gänsicke, B. T., Hagen, H.-J., et al. 2005b, A\&A, 440, 701 Rogoziecki, P., \& Schwarzenberg-Czerny, A. 2001, MNRAS, 323, 850

Roth, M. M. 1992, in CCDs in astronomy, ed. G. Jacoby, ASP Conf. Ser., 8, 380 Scargle, J. D. 1982, ApJ, 263, 835

Schneider, D. P., \& Young, P. 1980, ApJ, 238, 946

Schwarz, R., Schwope, A. D., \& Staude, A. 2001, A\&A, 374, 189

Schwarzenberg-Czerny, A. 1989, MNRAS, 241, 153

Schwarzenberg-Czerny, A. 1996, ApJ, 460, L107

Shafter, A. W. 1992, ApJ, 394, 268

Shafter, A. W., \& Holland, J. N. 2003, PASP, 115, 1105

Shafter, A. W., Wheeler, J. C., \& Cannizzo, J. K. 1986, ApJ, 305, 261

Spruit, H. C., \& Ritter, H. 1983, A\&A, 124, 267

Stauffer, J., Spinrad, H., \& Thorstensen, J. 1979, PASP, 91, 59

Szkody, P., \& Mateo, M. 1986, AJ, 92, 483

Szkody, P., Desai, V., \& Hoard, D. W. 2000, AJ, 119, 365

Tappert, C., \& Bianchini, A. 2003, A\&A, 401, 1101

Thorstensen, J. R., \& Fenton, W. H. 2002, PASP, 114, 74

Thorstensen, J. R., \& Taylor, C. J. 2001, MNRAS, 326, 1235

Thorstensen, J. R., Patterson, J., Kemp, J., \& Vennes, S. 2002, PASP, 114, 1108

Tovmassian, G. H., Greiner, J., Zharikov, S. V., Echevarría, J., \& Kniazev, A. 2001, A\&A, 380, 504

Townsley, D. M., \& Bildsten, L. 2003, ApJ, 596, L227

van Teeseling, A., Beuermann, K., \& Verbunt, F. 1996, A\&A, 315, 467

Voges, W., Aschenbach, B., Boller, T., et al. 1999, A\&A, 349, 389

Voges, W., Aschenbach, B., Boller, T., et al. 2000, IAU Circ., 7432

Wade, R. A. 1979, AJ, 84, 562

Warner, B. 1995, Cataclysmic Variable Stars (Cambridge: Cambridge University Press)

Wu, X., Li, Z., Gao, W., \& Leung, K. 2001, ApJ, 549, L81

Zhang, E.-H., \& Robinson, E. L. 1987, ApJ, 321, 813 
A. Aungwerojwit et al.: Dwarf novae in the Hamburg quasar survey: rarer than expected, Online Material $p 1$

\section{Online Material}


Table 2. Log of the observations.

\begin{tabular}{|c|c|c|c|c|c|}
\hline Date & Telescope & $\begin{array}{l}\text { Filter/ } \\
\text { Grism }\end{array}$ & $\begin{array}{c}\text { Exp. } \\
\text { (s) }\end{array}$ & Frames & $\begin{array}{c}\text { Comp. } \\
\text { star }\end{array}$ \\
\hline \multicolumn{6}{|l|}{ HS 0417+7445 } \\
\hline 1996 Oct. 05 & CA22 & $B-400$ & 600 & 1 & - \\
\hline 2000 Dec. 21 22:41-02:37 & WD & $B$ & 240 & 53 & $\mathrm{C} 1$ \\
\hline 2001 Jan. 14 00:40-04:40 & WD & $B$ & 240 & 54 & $\mathrm{C} 1$ \\
\hline 2003 Feb. 27 20:35-23:47 & OLT & $R$ & 300 & 37 & $\mathrm{C} 2$ \\
\hline 2004 Nov. 12 17:40-02:03 & WS & clear & 150 & 162 & $\mathrm{C} 2$ \\
\hline 2005 Jan. 03 02:18-04:40 & INT & $g^{\prime}$ & 80 & 71 & $\mathrm{C} 2$ \\
\hline 2005 Jan. 04 23:07-02:27 & INT & $g^{\prime}$ & $30-35$ & 162 & $\mathrm{C} 2$ \\
\hline \multicolumn{6}{|l|}{ HS 1016+3412 } \\
\hline 2001 Apr. 30 & CA22 & $B-200$ & 600 & 1 & - \\
\hline 2003 Apr. 07 & CA22 & $G-100$ & 600 & 1 & - \\
\hline 2003 Apr. 08 21:09-22:19 & CA22 & $G-100$ & 600 & 7 & - \\
\hline 2003 Apr. $10 \quad 22: 28$ & CA22 & $G-100$ & 600 & 1 & - \\
\hline 2003 Apr. 12 22:28-00:10 & CA22 & $G-100$ & 600 & 9 & - \\
\hline 2003 Apr. 24 22:07-23:01 & INT & R632V & 600 & 6 & - \\
\hline 2003 Apr. 25 23:31-00:24 & INT & R632V & 600 & 6 & - \\
\hline 2003 Apr. 27 22:27-23:28 & CA22 & $G-100$ & 600 & 6 & - \\
\hline 2003 Apr. 28 22:31-23:24 & INT & R632V & 600 & 6 & - \\
\hline 2003 May 18 22:08-22:51 & INT & R632V & 600 & 6 & - \\
\hline 2004 May 23 19:52-21:59 & KY & clear & $45-90$ & 104 & C3 \\
\hline 2004 May 24 20:09-21:47 & KY & clear & 60 & 55 & C3 \\
\hline 2004 May 26 19:51-22:20 & KY & clear & 45 & 157 & C3 \\
\hline 2004 May 27 19:58-23:10 & KY & clear & $60-75$ & 116 & C3 \\
\hline 2004 May 28 20:12-22:48 & KY & clear & 60 & 101 & C3 \\
\hline 2004 May 29 22:52-23:57 & IAC80 & clear & 125 & 29 & $\mathrm{C} 3$ \\
\hline 2004 May 30 22:55-23:58 & IAC80 & clear & 120 & 28 & C3 \\
\hline 2004 May 31 21:18-22:48 & IAC80 & clear & 113 & 45 & C3 \\
\hline 2005 Feb. 12 23:34-05:24 & CA22 & $G-100$ & 600 & 24 & $\mathrm{C} 3$ \\
\hline \multicolumn{6}{|l|}{ HS 1340+1524 } \\
\hline 2001 May 01 & CA22 & $B-200$ & 600 & 1 & - \\
\hline 2001 May 08 20:17-02:00 & AIP & $R$ & 120 & 200 & C6 \\
\hline 2001 May 09 20:51-00:23 & AIP & $R$ & 120 & 83 & C6 \\
\hline 2002 Jul. $02 \quad$ 18:57-22:42 & KY & $R$ & 120 & 86 & $\mathrm{C} 4$ \\
\hline 2002 Jul. $04 \quad 20: 02-22: 17$ & KY & $R$ & 120 & 58 & $\mathrm{C} 4$ \\
\hline 2003 Apr. 07 22:37-23:29 & CA22 & $G-100$ & 600 & 5 & - \\
\hline 2003 Apr. 08 22:42-23:41 & CA22 & $G-100$ & 600 & 6 & - \\
\hline 2003 Apr. 09 23:48-00:10 & CA22 & $G-100$ & 600 & 3 & - \\
\hline 2003 Apr. 10 22:46-00:29 & CA22 & $G-100$ & 600 & 7 & - \\
\hline 2003 Apr. 11 00:39-04:52 & CA22 & V & 30 & 179 & $\mathrm{C} 4$ \\
\hline 2003 Apr. 11 21:45-01:35 & CA22 & $G-100$ & 600 & 11 & - \\
\hline 2003 Apr. 13 00:31-03:28 & CA22 & $G-100$ & 600 & 12 & - \\
\hline 2003 Apr. 24 00:26-01:19 & INT & R632V & 600 & 6 & - \\
\hline 2003 Apr. 25 00:34-01:26 & INT & R632V & 600 & 6 & - \\
\hline 2003 Apr. 28 22:54-00:32 & CA22 & $G-100$ & 600 & 7 & - \\
\hline 2003 May 29 19:44-22:03 & KY & $R$ & 90 & 69 & $\mathrm{C} 4$ \\
\hline 2003 May 30 19:17-01:12 & $\mathrm{KY}$ & $R$ & 90 & 212 & $\mathrm{C} 4$ \\
\hline 2003 Jun. 24 19:28-22:08 & KY & $R$ & 90 & 101 & $\mathrm{C} 4$ \\
\hline 2003 Jun. 28 19:21-22:10 & KY & $R$ & 120 & 76 & $\mathrm{C} 4$ \\
\hline 2004 Mar. 17 07:18-11:38 & FLWO & clear & 40 & 297 & C5 \\
\hline
\end{tabular}

\begin{tabular}{lcccccc}
\hline \hline Date & UT & Telescope & $\begin{array}{c}\text { Filter/ } \\
\text { Grism }\end{array}$ & $\begin{array}{c}\text { Exp. } \\
\text { (s) }\end{array}$ & $\begin{array}{c}\text { Frames Comp. } \\
\text { star }\end{array}$ \\
\hline 2004 Mar. 18 & 07:23-12:00 & FLWO & clear & 40 & 273 & C5 \\
2004 May 19 23:14-03:01 & IAC80 & clear & 60 & 193 & C6 \\
2004 May 20 22:43-00:58 & IAC80 & clear & 90 & 78 & C6 \\
2004 May 22 21:39-00:34 & KY & clear & 30 & 262 & C4 \\
2004 Jun. 10 20:01-21:54 & KY & clear & 30 & 176 & C4 \\
2005 Feb. 18 & 01:13-05:10 & CA22 & $G-100$ & 600 & 15 & - \\
2005 May 11 23:10-03:04 & IAC80 & clear & $45-60$ & 203 & C6 \\
2005 May 12 22:41-04:20 & IAC80 & clear & 60 & 213 & C6 \\
2005 May 13 22:07-04:09 & IAC80 & clear & 60 & 270 & C6
\end{tabular}

\section{HS $1857+7127$}

1990 Jul. $31 \quad 02: 32 \quad$ CA22 $120 \AA / \mathrm{mm} 3600 \quad 1 \quad-$ 2002 Apr. 03 23:30-03:29 AIP $\quad R \quad 120 \quad 118 \quad$ C9 2002 Apr. 22 19:52-02:38 AIP $\quad R \quad 120 \quad 185 \quad$ C9

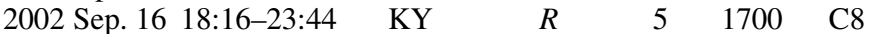
2002 Sep. 17 18:31-21:50 $\quad$ KY $\quad \begin{array}{lllll}R & 5-10 & 683 & \text { C8 }\end{array}$

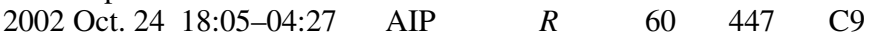
2003 Apr. 08 02:44-03:44 CA22 G-100 $600 \quad 6 \quad-$ 2003 Apr. 09 02:10-04:47 CA22 $G-100 \quad 600 \quad 10 \quad-$ 2003 Apr. 13 02:25-04:50 CA22 $G-100 \quad 600 \quad 9 \quad-$ 2003 Apr. 22 19:45-03:04 AIP $\quad R \quad 60 \quad 386 \quad$ C9 2003 Apr. 25 04:01-05:03 INT R632V $600 \quad 7 \quad-$ 2003 Apr. 27 05:32-05:43 INT R632V $600 \quad 2$ 2003 Apr. 29 04:18-04:30 CA22 $\quad G-100 \quad 600 \quad 2 \quad-$ 2003 Apr. 29 04:46-05:28 INT R632V $600 \quad 5$ -

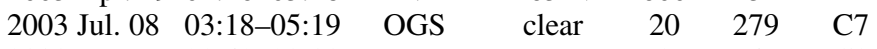
$\begin{array}{llllll}2003 \text { Aug. 17 22:42-23:08 KY } & V & 8 & 147 & \text { C8 }\end{array}$ 2003 Aug. $17 \quad 03: 28$ HST/STIS G140L $800 \quad 1 \quad-$ 2004 May 03 00:21-05:46 IAC80 clear $15 \quad 922 \quad$ C7

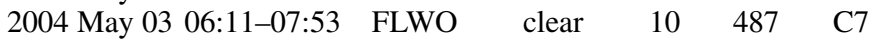
2004 May 04 08:25-11:15 FLWO clear $\quad 10 \quad 809 \quad$ C7

HS 2214+2845

2000 Sep. $20 \quad 21: 26$ 2000 Sep. 21 03:28-11:04 2000 Sep. 24 02:44-10:53 2000 Sep. 24 20:10-23:55 2000 Sep. 24 20:23-20:54 2002 Aug. 29 02:25-03:48 2002 Sep. 01 03:07-03:58 2002 Sep. 02 02:51-03:22 2002 Sep. 04 00:23-00:54 2003 Jun. 23 23:54-02:08 2003 Jun. 25 00:06-02:11 2003 Jun. 27 23:35-00:44 2003 Jun. 28 22:49-00:35 2003 Jul. 15 02:29-05:09 2003 Sep. 20 21:31-02:30 2003 Sep. 22 20:14-03:10 IAC80 2003 Sep. 24 00:16-03:59 IAC80

CA22
BS
BS
CA22
CA22
INT
INT
INT
INT
KY
KY
KY
KY
OGS
IAC80
IAC80
IAC80
IAC80

$\begin{array}{cccc}B-200 & 600 & 1 & - \\ R & 100 & 245 & : \\ R & 100 & 254 & : \\ B-100 & 600 & 16 & - \\ R-100 & 600 & 2 & - \\ \mathrm{R} 632 \mathrm{~V} & 600 & 9 & - \\ \mathrm{R} 632 \mathrm{~V} & 600 & 6 & - \\ \mathrm{R} 632 \mathrm{~V} & 600 & 4 & - \\ \mathrm{R} 632 \mathrm{~V} & 600 & 4 & - \\ R & 90 & 85 & \mathrm{C} 11 \\ R & 90 & 80 & \mathrm{C} 11 \\ R & 30 & 113 & \mathrm{C} 11 \\ R & 60 & 93 & \mathrm{C} 11 \\ \text { clear } & 15 & 442 & \mathrm{C} 10 \\ \text { clear } & 10 & 871 & \mathrm{C} 10 \\ \text { clear } & 10 & 1187 & \mathrm{C} 10 \\ \text { clear } & 10 & 772 & \mathrm{C} 10 \\ \text { clear } & 10 & 689 & \mathrm{C} 10\end{array}$

Notes. CA22: $2.2 \mathrm{~m}$ telescope at Calar Alto Observatory, using CAFOS with a 2k $\times 2 \mathrm{k}$ SITe pixel CCD; WS: $0.8 \mathrm{~m}$ telescope at Wendelstein Observatory, using the MONICA CCD camera (Roth 1992); OLT: $1.2 \mathrm{~m}$ Oskar Lühning Teleskop at Hamburg Observatory, equipped with a $1 \mathrm{k} \times 1 \mathrm{k}$ pixel SITe CCD; INT: $2.5 \mathrm{~m}$ Isaac Newton Telescope on Observatorio del Roque de los Muchachos, equipped with the Wide Field Camera (WFC), an array of four EEV 2k $\times 4 \mathrm{k}$ pixel CCDs; KY: $1.2 \mathrm{~m}$ telescope at Kryoneri Observatory, using a Photometrics SI-502 $516 \times 516$ pixel CCD camera; IAC80: $0.82 \mathrm{~m}$ telescope at Observatorio del Teide, equipped with Thomson $1 \mathrm{k} \times 1 \mathrm{k}$ pixel CCD camera; OGS: $1 \mathrm{~m}$ Optical Ground Station at Observatorio del Teide, equipped with Thomson $1 \mathrm{k} \times 1 \mathrm{k}$ pixel CCD camera; AIP: $0.7 \mathrm{~m}$ telescope of the Astrophysikalisches Institut Potsdam, using with $1 \mathrm{k} \times 1 \mathrm{k}$ pixel SITe CCD; FLWO: $1.2 \mathrm{~m}$ telescope at Fred Lawrence Whipple Observatory, equipped with the 4-Shooter CCD camera, an array of four $2 \mathrm{k} \times 2 \mathrm{k}$ pixel, and only a small part of the CCD\#3 was read out; BS: $0.41 \mathrm{~m}$ at Braeside Observatory telescope, using a SITe $512 \times 512$ pixel CCD camera; the comparison stars used in instrumental magnitude extractions from Braeside are unknown (marked by colons). 
A. Aungwerojwit et al.: Dwarf novae in the Hamburg quasar survey: rarer than expected, Online Material p 3
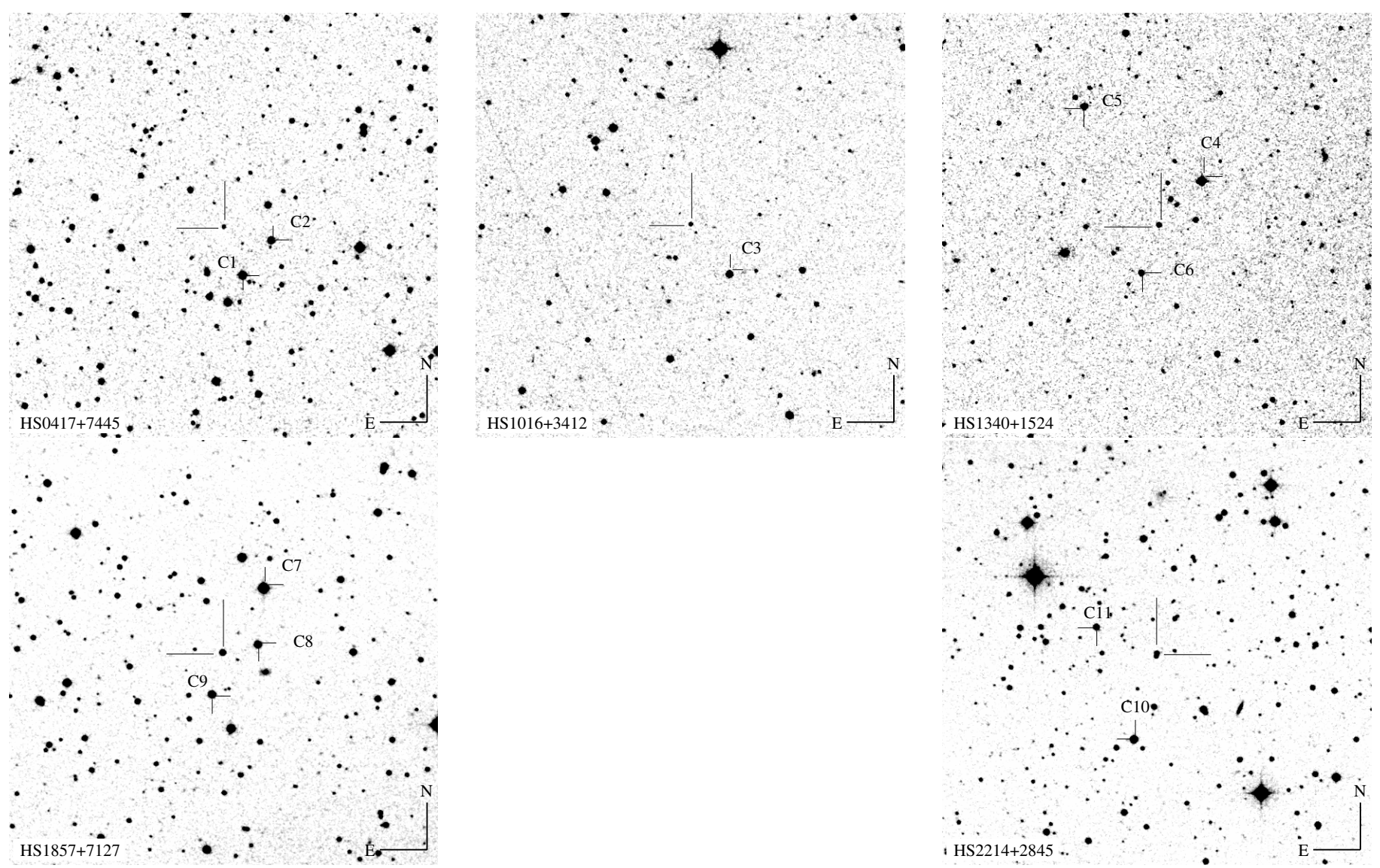

Fig. 1. $10^{\prime} \times 10^{\prime}$ finding charts for HS 0417, HS 1016, HS 1340, HS 1857, and HS 2214 obtained from the Digitized Sky Survey. HS 2214 is the northern component of a close visual binary. See Table 3 for details on the comparison stars C1-C11.

Table 3. Comparison stars used for the differential CCD photometry of HS 0417, HS 1016, HS 1340, HS 1857, and HS 2214 , see Fig. 1.

\begin{tabular}{cccc}
\hline \hline ID & USNO-A2.0 & $R$ & $B$ \\
\hline C1 & $1575-02009718$ & 13.3 & 13.3 \\
C2 & $1575-02008711$ & 13.6 & 14.9 \\
C3 & $1200-06495553$ & 14.3 & 15.0 \\
C4 & $1050-06991669$ & 13.4 & 14.5 \\
C5 & $1050-06992410$ & 14.4 & 16.4 \\
C6 & $1050-06992029$ & 15.3 & 17.3 \\
C7 & $1575-04072972$ & 12.2 & 13.5 \\
C8 & $1575-04073098$ & 13.8 & 14.8 \\
C9 & $1575-04073991$ & 13.8 & 14.9 \\
C10 & $1125-19198939$ & 13.7 & 14.8 \\
C11 & $1125-19199670$ & 15.0 & 15.6 \\
\hline
\end{tabular}

